\title{
Symmetric Complex Boundary Element Scheme for 2-D Stokes Mixed Boundary Value Problem
}

\author{
Sun-Gwon Hong \\ Institute of Mathematics, \\ Academy of Sciences, \\ Pyongyang, DPR Korea \\ e-mail: math.inst@star-co.net.kp
}

\begin{abstract}
For 2-D Stokes mixed boundary value problems we construct a boundary integral equation which couples a conventional boundary integral equation for the velocity with a hypersingular boundary integral equation for the traction. Expressing terms in the equation by complex variables, we obtain a complex boundary integral equation and realize symmetrization of boundary element scheme by Galerkin method. Applying a boundary limit method, we obtain exact calculation formulae for calculation of hypersingular boundary integrals. It is shown that all divergent terms in hypersingular integrals cancel each other out.
\end{abstract}

Key words: hypersingular integral, boundary limit method, complex boundary element method, symmetric boundary element scheme.

AMS 2000 subject classification: 65M38, 65P30, 76M15, 78M15,

\section{Introduction}

Boundary element method(BEM) allows us to get approximate solutions of problems of mathematical physics on a domain $\Omega$ in $\mathbb{R}^{d}(d=2,3)$ by the solution of boundary integral equations corresponding to the problems.

For large-scale problems, if the final linear systems are symmetric, then iterative methods are useful, which can reduce the required computer memory and has a good convergence property. In view of this for many problems including potential problems and elastic one, the symmetrization of boundary element scheme is widely considered([19]). For example, to get a boundary integral equation for symmetric boundary element scheme to solve potential problems with mixed boundary conditions, first one express the solution on $\Omega$, potential, by the sum of simple layer and double layer potentials, densities of which are boundary flux and boundary potential. Then, by taking limit to boundary, one get the "potential boundary integral equation". Next, getting the directional derivative of the expression of the potential and limiting to the boundary again, one get another boundary integral 
equation- "flux boundary integral equation". The flux boundary integral equation contains a potential adjoint to the double layer potential and a hypersingular boundary potential $([19])$.

In the case of Dirichlet boundary value problem, from the potential boundary integral equation one can get a simple layer potential operator equation with the unknown boundary flux. The simple layer potential operator is self-adjoint, and so Galerkin discretization of the equation is symmetric. In the case of mixed boundary conditions the velocity boundary integral equation includes not only simple layer potential operator, but also a double layer potential operator which is not self-adjoint, and so it is difficult to get symmetric discrete scheme from the equation. Therefore, by combination of the potential boundary integral equation with the flux boundary integral equation symmetrizations of conventional potential problems $([1,17])$ are realized. The flux boundary integral equation contains hypersingular integral, so, it is important to get exact values of these hypersingular integrals $([9])$.

For calculation of hypersingular integrals Hadamard's finite part integral is usually used. To apply Hadamard's finite part integral, basis functions which become density functions must be in at least $C^{1, \alpha}$-class, but piecewise linear continuous basis used widely in boundary element scheme belong to $C^{0,1}$-class. Such situations led to studies of methods to weaken the singularity of integrals $([3,4])$ and the smoothness requirement of density functions $([16,20])$ and to get numerical values $([6,12,18])$.

In [19], in particular, as a method to calculate hypersingular integral in boundary element schemes unlike to Hadamard finite part integral method, the boundary limit method was suggested. Main procedure of the boundary limit method is as follows. First, with source point off the boundary, one integrates. Then taking limit of the result as the point goes to the boundary, one extracts a converging finite part to be regarded as an integral value and check whether all divergent terms are canceled. Authors mentioned that it is an advantage of the boundary limit method that it can obtain the value of hypersingular integral even when density function is not smooth, as piecewise linear continuous basis function, and deal with arbitrary integrals with singularity by the same way.

But, for mixed boundary value problems of the Stokes equation there is no such results-symmetrizations and evaluation of hypersingular integrals, yet.

On the other hand, the boundary element method works, commonly, in real variables.

For two dimensional vector-value problems such as 2-D Stokes flow problem, however, to consider complex variables has advantage that enable to avoid complexity due to matrix-vector operation.

Complex boundary element methods were studied in $[13,14,21]$ for potential problems, in [5] for Helmholtz equation, in [8] for electrochemical system, in [10] for flow around obstacles and in [15] for linear elastostatic problems.

Using the formulae in [15] for a linear elastic problem, [14] studied complex potential expressions for 2-D Stokes flow problems. But, the results are not for symmetric scheme. 
In this paper, we are concerned with symmetric complex boundary element scheme for 2-D Stokes equation with velocity and traction boundary conditions together. Following the ideas for potential and elastic problems above mentioned, we have a real boundary integral equation to get symmetric boundary element scheme. Expressing variables in the real boundary integral equation by complex variables, we obtain the complex boundary integral equation. We construct a discrete scheme by Galerkin method. And assuming existence of hypersingular complex boundary integrals on discrete boundary, we prove the convergence and stability of the scheme. Furthermore, applying the boundary limit method in [19] and proving that all divergent terms are canceled, we get formulae for calculation of all hypersingular complex boundary integrals.

This paper consists of 5 sections.

In Section 2, combining a "velocity boundary integral equation" and a "traction one" on the hole boundary, we get a system of "velocity equation" on the portion for velocity boundary condition and "traction equation" on the portion for traction boundary condition, and apply Galerkin scheme to get symmetric boundary element scheme. Then, 5 basic terms are expressed by complex variables and using them, complex expressions of boundary potentials are obtained(Theorem 2.1). After that, a complex boundary element scheme is constructed by using piecewise linear continue elements for the velocity and piecewise constant elements for the traction. Symmetry, convergence and stability of the scheme are proved(Theorem $2.2)$.

In Section 3, by the boundary limit method we get the exact formulae for calculation of hypersingular complex boundary integrals in the scheme and prove cancelation of all divergent terms(Theorem 3.1-3.5).

In Section 4 an algorithm to calculate all elements excluding hypersingular boundary integral in coefficient matrix of the complex discrete equation and elements in right-hand side of the equation.

In Section 5, results of the paper are summarized.

Finally, many proofs and calculations which make the reading of the paper difficult are collected in Appendix.

\section{Symmetric complex boundary element scheme}

\subsection{Stokes mixed boundary value problem}

Let us consider the following mixed boundary value problem of the Stokes equation.

$$
\begin{aligned}
& -\mu \Delta u+\nabla p=0, \quad \operatorname{div} u=0, \quad x \in \Omega \subset \mathbb{R}^{2}, \\
& u=f, \quad x \in \Gamma_{1} \text {, } \\
& T(u)=g, \quad x \in \Gamma_{2} \text {. }
\end{aligned}
$$

Here $\Omega$ is a simply connected bounded domain and boundary $\Gamma=\bar{\Gamma}_{1} \cup \bar{\Gamma}_{2}$ is smooth, $u$ - the velocity, $p$ - the pressure, $\mu$ - the dynamical viscocity coefficient, $f$ 
and $g$ are, respectively, traces of velocity $u$ and $\operatorname{traction} T(u)$ on $\Gamma_{1}$ and $\Gamma_{2}$, and $n$ is outward outward normal unit vector on the boundary of domain.

Traction is given by

$$
T(u):=-p n+\mu\left(\nabla u+\nabla u^{T}\right) n
$$

and it's adjoint traction is defined by

$$
T^{\prime}(u):=p n+\mu\left(\nabla u+\nabla u^{T}\right) n .
$$

Let $E(x, y)$ be the fundamental solution(matrix) to the Stokes operator. Define

$$
\begin{aligned}
& F(x, y):=T_{y}^{\prime}(E(x, y))^{T}, \\
& G(x, y):=T_{x}(E(x, y)), \\
& H(x, y):=-T_{x} T_{y}^{\prime}(E(x, y))^{T} .
\end{aligned}
$$

Denote by $V, K, K^{\prime}$ and $D$, respectively, boundary integral operators of which kernels are, respectively, $E(x, y), F(x, y), G(x, y)$ and $H(x, y)$. Then, by the boundary integral operators we have the following continuous mappings $([11])$.

$$
\begin{array}{ll}
V: H^{-\frac{1}{2}}(\Gamma) \rightarrow H^{\frac{1}{2}}(\Gamma), & K: H^{\frac{1}{2}}(\Gamma) \rightarrow H^{\frac{1}{2}}(\Gamma), \\
K^{\prime}: H^{-\frac{1}{2}}(\Gamma) \rightarrow H^{-\frac{1}{2}}(\Gamma), & D: H^{\frac{1}{2}}(\Gamma) \rightarrow H^{-\frac{1}{2}}(\Gamma) .
\end{array}
$$

With tensor notation, kernel functions can be written as follows $([11,15])$.

$$
\begin{aligned}
E_{i, j}(x, y) & =\frac{1}{4 \pi \mu}\left(\delta_{i j} \log \frac{1}{r}+r_{, i} r_{, j}-\frac{1}{2} \delta_{i j}\right), \\
F_{i, j}(x, y) & =-\frac{1}{\pi r}\left(r_{, i} r_{, j} r_{, k} n_{k}(y)\right), \\
G_{i, j}(x, y) & =\frac{1}{\pi r}\left(r_{, i} r_{, j} r_{, k} n_{k}(x)\right), \\
H_{i, j}(x, y) & =\frac{\mu}{\pi r^{2}}(x, y)=\frac{\mu}{\pi r^{2}}\left[\left(\delta_{i j} r_{, k} r_{, k}\right) r_{, l} n_{l}(y)\right. \\
& \left.+n_{i}(y) r_{, j} r_{, k}+n_{k}(y) r_{, i} r_{, j}+\delta_{i k} n_{j}\right] n_{k}(x),
\end{aligned}
$$

where $r=|x-y|, r_{, j}=\partial r / \partial y_{j}$ and Einstein convention for sub-indices is used. Constant term $-\frac{1}{2} \delta_{i j}$ in expression (6), which does not affect the solution, is added for convenience in the complex variable expression.

Putting $\left.u\right|_{\Gamma}=: \varphi,\left.T(u)\right|_{\Gamma}=: \tau$, we obtain boundary integral equation([11])

$$
\begin{array}{ll}
\frac{1}{2} \varphi(x)=V \tau(x)-K \varphi(x), & x \in \Gamma, \\
\frac{1}{2} \tau(x)=K^{\prime} \tau(x)+D \varphi(x), & x \in \Gamma .
\end{array}
$$


Let us introduce functional spaces as follows.

$$
\begin{aligned}
& s \geq 0: H^{s}\left(\Gamma_{i}\right):=\left\{\left.v\right|_{\Gamma_{i}}: v \in H^{s}(\Gamma)\right\} \\
& \widetilde{H}^{s}\left(\Gamma_{i}\right):=\text { complete }\left\{v \in H^{s}(\Gamma): \operatorname{supp}(v) \subset \Gamma_{i}\right\}, \\
& s<0: H^{s}\left(\Gamma_{i}\right):=\left(H_{0}^{-s}\left(\Gamma_{i}\right)\right)^{\prime}, \quad \widetilde{H}^{s}\left(\Gamma_{i}\right):=\left(\widetilde{H}^{-s}\left(\Gamma_{i}\right)\right)^{\prime} .
\end{aligned}
$$

Denote $H^{s}\left(\Gamma_{j}\right) \rightarrow H^{s}\left(\Gamma_{i}\right)$ - contractions of the operators $V, K, K^{\prime}$ and $D$, respectively, by $V_{i, j}, K_{i, j}, K_{i, j}^{\prime}$ and $D_{i, j}$.

Setting $\left.T(u)\right|_{\Gamma_{1}}=w,\left.u\right|_{\Gamma_{2}}=\phi$ and taking into account the boundary condition of the first boundary integral equation at $x \in \Gamma_{1}$ and one of the second equation at $x \in \Gamma_{2}$, we get the following boundary integral equation

$$
\begin{aligned}
& \frac{1}{2} f(x)+K_{1,1} f(x)+K_{1,2} \phi(x)-V_{1,1} w(x)-V_{1,2} g(x)=0, \quad x \in \Gamma_{1}, \\
& \frac{1}{2} g(x)-K_{2,1}^{\prime} w(x)-K_{2,2}^{\prime} g(x)-D_{2,1} f(x)-D_{2,2} \phi(x)=0, \quad x \in \Gamma_{2},
\end{aligned}
$$

which is written as follow.

$$
\left[\begin{array}{cc}
V_{1,1} & -K_{1,2} \\
K_{2,1}^{\prime} & D_{2,2}
\end{array}\right]\left[\begin{array}{c}
w \\
\phi
\end{array}\right]=\left[\begin{array}{cc}
-V_{1,2} & 1 / 2 I_{1}+K_{1,1} \\
1 / 2 I_{2}-K_{2,2}^{\prime} & -D_{2,1}
\end{array}\right]\left[\begin{array}{l}
g \\
f
\end{array}\right]
$$

where $I_{i}, i=1,2$ are ,respectively, identity in $\Gamma_{i}$.

Let

$$
V_{\Gamma}:=H^{-\frac{1}{2}}(\Gamma) \times H^{\frac{1}{2}}(\Gamma), \quad \widetilde{V}_{\Gamma}:=\widetilde{H}^{-\frac{1}{2}}\left(\Gamma_{1}\right) \times \widetilde{H}^{\frac{1}{2}}\left(\Gamma_{2}\right)
$$

and rewrite $\left[\begin{array}{c}\tau \\ \varphi\end{array}\right] \in V_{\Gamma}$ as

$$
\left[\begin{array}{l}
\tau \\
\varphi
\end{array}\right]=\left[\begin{array}{l}
\tau^{0} \\
\varphi^{0}
\end{array}\right]+\left[\begin{array}{l}
\widehat{\tau} \\
\widehat{\varphi}
\end{array}\right], \quad\left[\begin{array}{l}
\tau^{0} \\
\varphi^{0}
\end{array}\right] \in \widetilde{V}_{\Gamma}, \quad\left[\begin{array}{l}
\widehat{\tau} \\
\widehat{\varphi}
\end{array}\right] \in V_{\Gamma} .
$$

Define a bilinear form

$$
a\left(\left[\begin{array}{c}
\tau^{\prime} \\
\varphi^{\prime}
\end{array}\right],\left[\begin{array}{l}
\xi \\
p
\end{array}\right]\right):=\left\langle\left[\begin{array}{cc}
V & -K \\
K^{\prime} & D
\end{array}\right]\left[\begin{array}{l}
\tau^{\prime} \\
\varphi^{\prime}
\end{array}\right],\left[\begin{array}{l}
\xi \\
p
\end{array}\right]\right\rangle, \quad \widetilde{V}_{\Gamma} \times \widetilde{V}_{\Gamma} \rightarrow \mathbb{R}
$$

Then, by (10) we have

$$
a\left(\left[\begin{array}{l}
\tau^{0} \\
\varphi^{0}
\end{array}\right],\left[\begin{array}{l}
\xi \\
p
\end{array}\right]\right)=b\left(\left[\begin{array}{l}
\xi \\
p
\end{array}\right]\right), \quad \forall\left[\begin{array}{l}
\xi \\
p
\end{array}\right] \in \widetilde{V}_{\Gamma}
$$

where

$$
b\left(\left[\begin{array}{l}
\xi \\
p
\end{array}\right]\right):=\left\langle\left[\begin{array}{cc}
-V & 1 / 2 I+K \\
1 / 2 I-K^{\prime} & -D
\end{array}\right]\left[\begin{array}{l}
\widehat{\tau} \\
\widehat{\varphi}
\end{array}\right],\left[\begin{array}{l}
\xi \\
p
\end{array}\right]\right\rangle .
$$


Now, we convert real vector potentials $V \tau(x), K \varphi(x), K^{\prime} \tau(x)$ and $D \varphi(x)$ to complex variable forms. To this end, put

$$
\begin{aligned}
& \varsigma:=\left.\left(x_{1}+i x_{2}\right)\right|_{\Gamma}, z:=\left.\left(y_{1}+i y_{2}\right)\right|_{\Gamma}, n(z)=\left.\left(n_{1}(y)+i n_{2}(y)\right)\right|_{\Gamma}, \\
& \varphi(z):=\varphi_{1}(y)+i \varphi_{2}(y), \tau(z):=\tau_{1}(y)+i \tau_{2}(y) .
\end{aligned}
$$

Then, we have that

$$
x_{1}-y_{1}=\frac{1}{2}(\varsigma-z+\overline{\varsigma-z}), x_{2}-y_{2}=\frac{1}{2 i}(\varsigma-z-\overline{\varsigma-z})
$$

and components of vector value function $\left(u_{1}(y), u_{2}(y)\right)^{T}$ are as follows.

$$
u_{1}(y)=\frac{1}{2}(u(z)+\overline{u(z)}), u_{2}(y)=\frac{1}{2 i}(u(z)-\overline{u(z)}) .
$$

Also we have the following equalities which are proved in A1 of the Appendix.

(i) $r_{, j}(x, y) u_{j}(y)=-\frac{1}{2 r}((\varsigma-z) \overline{u(z)}+(\bar{\varsigma}-\bar{z}) u(z))$,

(ii) $n_{j}(y) u_{j}(y)=\frac{1}{2}(n(z) \overline{u(z)}+\overline{n(z)} u(z))$,

(iii) $r_{, j}(x, y) n_{j}(x)=-\frac{1}{2 r}((\varsigma-z) \overline{n(\varsigma)}+(\bar{\varsigma}-\bar{z}) n(\varsigma))$,

(iv) $r_{, j}(x, y) n_{j}(y)=-\frac{1}{2 r}((\varsigma-z) \overline{n(z)}+(\bar{\varsigma}-\bar{z}) n(z))$,

(v) $r_{, i}(x, y) r_{, j}(x, y) u_{j}(y)=\frac{1}{2}\left(\frac{\varsigma-z}{\bar{\varsigma}-\bar{z}} \overline{u(z)}+u(z)\right)$.

Theorem 2.1 The followings equalities hold.

$$
\begin{aligned}
& \int_{\Gamma} E(x, y) \tau(y) d \Gamma(y)=\int_{\Gamma} E_{1, j}(x, y) \tau_{j}(y) d \Gamma(y)+i \int_{\Gamma} E_{2, j}(x, y) \tau_{j}(y) d \Gamma(y) \\
& =\frac{1}{8 \pi \mu} \int_{\Gamma}\left(\log \frac{1}{\varsigma-z} \tau(z)+\log \frac{1}{\bar{\varsigma}-\bar{z}} \tau(z)+\frac{\varsigma-z \bar{\varsigma}-\bar{z}}{\tau(z)}\right) d \Gamma(z), \\
& \int_{\Gamma} F(x, y) \varphi(y) d \Gamma(y)=\int_{\Gamma} F_{1, j}(x, y) \varphi_{j}(y) d \Gamma(y)+i \int_{\Gamma} F_{2, j}(x, y) \varphi_{j}(y) d \Gamma(y) \\
& =\frac{1}{4 \pi} \int_{\Gamma}\left(\frac{1}{\varsigma-z} n(z) \varphi(z)+\frac{\varsigma-z}{(\bar{\varsigma}-\bar{z})^{2}} \overline{n(z) \varphi(z)}\right. \\
& \left.+\frac{1}{\bar{\varsigma}-\bar{z}}(n(z) \overline{\varphi(z)}+\overline{n(z)} \varphi(z))\right) d \Gamma(z), \\
& \int_{\Gamma} G(x, y) \tau(y) d \Gamma(y)=\int_{\Gamma} G_{1, j}(x, y) \tau_{j}(y) d \Gamma(y)+i \int_{\Gamma} G_{2, j}(x, y) \tau_{j}(y) d \Gamma(y) \\
& \quad=-\frac{1}{4 \pi} \int_{\Gamma}\left(\frac{1}{\varsigma-z} \tau(z)+\frac{1}{\bar{\varsigma}-\bar{z}} \overline{\tau(z)}\right) n(\varsigma) \\
& \left.+\left(\frac{1}{\bar{\varsigma}-\bar{z}} \tau(z)+\frac{\varsigma-z}{(\bar{\varsigma}-\bar{z})^{2}} \overline{\tau(z)}\right) n(\varsigma)\right) d \Gamma(z),
\end{aligned}
$$




$$
\begin{aligned}
\int_{\Gamma} & H(x, y) \varphi(y) d \Gamma(y)=\int_{\Gamma} H_{1, j}(x, y) \varphi_{j}(y) d \Gamma(y)+i \int_{\Gamma} H_{2, j}(x, y) \varphi_{j}(y) d \Gamma(y) \\
\quad & -\frac{\mu}{2 \pi} \int_{\Gamma}\left(\left(\frac{1}{(\varsigma-z)^{2}} n(z) \varphi(z)+\frac{1}{(\bar{\varsigma}-\bar{z})^{2}} \overline{n(z) \varphi(z)}\right) n(\varsigma)\right. \\
& \left.+\left(\frac{1}{(\bar{\varsigma}-\bar{z})^{2}}(n(z) \overline{\varphi(z)}+\overline{n(z)} \varphi(z))+\frac{2(\varsigma-z)}{(\bar{\varsigma}-\bar{z})^{3}} \overline{n(z) \varphi(z)}\right) \overline{n(\varsigma)}\right) d \Gamma(z) .
\end{aligned}
$$

\section{Proof.}

Now using formulae of kernel functions (6)-(9) and equalities(i)-(v), we have that

$$
\begin{aligned}
& E_{i, j}(x, y) \tau_{j}(y)= \\
& =\frac{1}{4 \pi \mu}\left(\tau(z) \frac{1}{2} \log \frac{1}{(\varsigma-z)(\bar{\varsigma}-\bar{z})}+\frac{1}{2}\left(\frac{\varsigma-z}{\bar{\varsigma}-\bar{z}} \overline{\tau(z)}+\tau(z)\right)-\frac{\tau(z)}{2}\right) \\
& =\frac{1}{8 \pi \mu}\left(\log \frac{1}{\varsigma-z} \tau(z)+\log \frac{1}{\bar{\varsigma}-\bar{z}} \tau(z)+\frac{\varsigma-z}{\bar{\varsigma}-\bar{z}} \overline{\tau(z)}\right) \text {, } \\
& F_{i, j}(, y) \varphi_{j}(y)= \\
& =-\frac{1}{\pi r} \frac{1}{2}\left(\frac{\varsigma-z}{\bar{\varsigma}-\bar{z}} \overline{\varphi(z)}+\varphi(z)\right) \frac{-1}{2|\varsigma-z|}((\varsigma-z) \overline{n(z)}+(\bar{\varsigma}-\bar{z}) n(z)) \\
& =\frac{1}{4 \pi}\left(\frac{\varsigma-z}{\bar{\varsigma}-\bar{z}} \overline{\varphi(z)}+\varphi(z)\right) \frac{1}{|\varsigma-z|^{2}}((\varsigma-z) \overline{n(z)}+(\bar{\varsigma}-\bar{z}) n(z)) \\
& =\frac{1}{4 \pi}\left(\frac{\varsigma-z}{\bar{\varsigma}-\bar{z}} \overline{\varphi(z)}+\varphi(z)\right)\left(\frac{\overline{n(z)}}{\bar{\varsigma}-\bar{z}}+\frac{n(z)}{\varsigma-z}\right) \\
& =\frac{1}{4 \pi}\left(\frac{n(z) \varphi(z)}{\varsigma-z}+\frac{1}{\bar{\varsigma}-\bar{z}}(n(z) \overline{\varphi(z)}+\overline{n(z)} \varphi(z))+\frac{\varsigma-z}{(\bar{\varsigma}-\bar{z})^{2}} \overline{n(z) \varphi(z)}\right), \\
& G_{i, j}(x, y) \tau_{j}(y)= \\
& =\frac{1}{\pi r} \frac{1}{2}\left(\frac{\varsigma-z}{\overline{\varsigma-z}} \overline{\tau(z)}+\tau(z)\right) \frac{-1}{2 r}((\varsigma-z) \overline{n(\varsigma)}+(\overline{\varsigma-z}) n(\varsigma)) \\
& =\frac{-1}{4 \pi}\left(\frac{n(\varsigma) \tau(z)}{\varsigma-z}+\frac{1}{\bar{\varsigma}-\bar{z}}(n(\varsigma) \overline{\tau(z)}+\overline{n(\varsigma)} \tau(z))+\frac{\varsigma-z}{(\bar{\varsigma}-\bar{z})^{2}} \overline{n(\varsigma) \tau(z)}\right) .
\end{aligned}
$$

Let us rewrite the (9) as follows

$$
\begin{aligned}
& H_{i, j}(x, y) \varphi_{j}(y)=\frac{\mu}{\pi r^{2}}\left[\varphi_{i} r_{, k} \mathrm{n}_{k} r_{, l} n_{l}+r_{, i} \varphi_{k} \mathrm{n}_{k} r_{, l} n_{l}\right. \\
& \left.\quad-8 r_{, i} r_{, j} \varphi_{j} r_{, k} \mathrm{n}_{k} r_{, l} n_{l}+n_{i} r_{, j} \varphi_{j} r_{, k} \mathrm{n}_{k}+r_{, i} n_{k} \mathrm{n}_{k} r_{, j} \varphi_{j}+\mathrm{n}_{i} n_{j} \varphi_{j}\right] .
\end{aligned}
$$

By (i)-(v) terms in [ ] of the right hand side are expressed as follows. 


$$
\begin{aligned}
& \varphi_{i} r_{, k} n_{k} r_{, l} n_{l}= \\
& =\frac{-\varphi(z)}{2 r}((\bar{\varsigma}-\bar{z}) n(\varsigma)+(\varsigma-z) \overline{n(\varsigma)}) \frac{-1}{2 r}((\bar{\varsigma}-\bar{z}) n(z)+(\varsigma-z) \overline{n(z)}) \\
& =\frac{n(\varsigma)}{4 r^{2}}((\bar{\varsigma}-\bar{z})(\bar{\varsigma}-\bar{z}) \varphi(z) n(z)+(\varsigma-z)(\bar{\varsigma}-\bar{z}) \varphi(z) \overline{n(z)}) \\
& +\frac{\overline{n(\varsigma)}}{4 r^{2}}((\varsigma-z)(\bar{\varsigma}-\bar{z}) \varphi(z) n(z)+(\varsigma-z)(\varsigma-z) \varphi(z) \overline{n(z)}), \\
& r_{, i} \varphi_{k} n_{k} r_{, l} n_{l}= \\
& =\frac{-(\varsigma-z)}{2 r}(\overline{\varphi(z)} n(\varsigma)+\varphi(z) \overline{n(\varsigma)}) \frac{-1}{2 r}((\bar{\varsigma}-\bar{z}) n(z)+(\varsigma-z) \overline{n(z)}) \\
& =\frac{n(\varsigma)}{4 r^{2}}((\varsigma-z)(\bar{\varsigma}-\bar{z}) \overline{\varphi(z)} n(z)+(\varsigma-z)(\varsigma-z) \overline{\varphi(z) n(z)}) \\
& +\frac{\overline{n(\varsigma)}}{4 r^{2}}((\varsigma-z)(\bar{\varsigma}-\bar{z}) \varphi(z) n(z)+(\varsigma-z)(\varsigma-z) \varphi(z) \overline{n(z)}), \\
& -8 r_{, i} r_{, j} \varphi_{j} r_{, k} n_{k} r_{, l} n_{l}=8 \frac{\varsigma-z}{r} \frac{-1}{2 r}((\varsigma-z) \overline{\varphi(z)}+(\bar{\varsigma}-\bar{z}) \varphi(z)) \\
& \times \frac{-1}{2 r}((\bar{\varsigma}-\bar{z}) n(\varsigma)+(\varsigma-z) \overline{n(\varsigma)}) \frac{-1}{2 r}((\bar{\varsigma}-\bar{z}) n(z)+(\varsigma-z) \overline{n(z)}) \\
& =\frac{-1}{4 r^{2}} n(\varsigma) \frac{4}{r^{2}}\left((\varsigma-z)^{2} \overline{\varphi(z)}+(\varsigma-z)(\bar{\varsigma}-\bar{z}) \varphi(z)\right) \\
& \times\left((\bar{\varsigma}-\bar{z})^{2} n(z)+(\bar{\varsigma}-\bar{z})(\varsigma-z) \overline{n(z)}\right) \\
& +\frac{-1}{4 r^{2}} \overline{n(\varsigma)} \frac{4}{r^{2}}\left((\varsigma-z)^{2} \overline{\varphi(z)}+(\varsigma-z)(\bar{\varsigma}-\bar{z}) \varphi(z)\right) \\
& \times\left((\varsigma-z)(\bar{\varsigma}-\bar{z}) n(z)+(\varsigma-z)^{2} \overline{n(z)}\right) \\
& =\frac{-1}{4 r^{2}} n(\varsigma) 4\left(\frac{\varsigma-z}{\bar{\varsigma}-\bar{z}} \overline{\varphi(z)}+\varphi(z)\right)\left((\bar{\varsigma}-\bar{z})^{2} n(z)+(\bar{\varsigma}-\bar{z})(\varsigma-z) \overline{n(z)}\right) \\
& +\frac{-1}{4 r^{2}} \overline{n(\varsigma)} 4\left(\frac{\varsigma-z}{\bar{\varsigma}-\bar{z}} \overline{\varphi(z)}+\varphi(z)\right)\left((\varsigma-z)(\bar{\varsigma}-\bar{z}) n(z)+(\varsigma-z)^{2} \overline{n(z)}\right) \text {, } \\
& n_{i} r_{, j} \varphi_{j} r_{, k} n_{k}= \\
& =\frac{-n(z)}{2 r}((\varsigma-z) \overline{\varphi(z)}+(\bar{\varsigma}-\bar{z}) \varphi(z)) \frac{-1}{2 r}((\bar{\varsigma}-\bar{z}) n(\varsigma)+(\varsigma-z) \overline{n(\varsigma)}) \\
& =\frac{n(\varsigma)}{4 r^{2}}((\varsigma-z)(\bar{\varsigma}-\bar{z}) \overline{\varphi(z)} n(z)+(\bar{\varsigma}-\bar{z})(\bar{\varsigma}-\bar{z}) \varphi(z) n(z)) \\
& +\frac{\overline{n(\varsigma)}}{4 r^{2}}((\varsigma-z)(\varsigma-z) \overline{\varphi(z)} n(z)+(\varsigma-z)(\bar{\varsigma}-\bar{z}) \varphi(z) n(z)),
\end{aligned}
$$




$$
\begin{aligned}
& r_{, i} n_{k} n_{k} r_{, j} \varphi_{j}= \\
& =\frac{z-\varsigma}{2 r}(\overline{n(z)} n(\varsigma)+n(z) \overline{n(\varsigma)}) \frac{-1}{2 r}((\varsigma-z) \overline{\varphi(z)}+(\bar{\varsigma}-\bar{z}) \varphi(z)) \\
& =\frac{n(\varsigma)}{4 r^{2}}((\varsigma-z)(\varsigma-z) \overline{\varphi(z) n(z)}+(\varsigma-z)(\bar{\varsigma}-\bar{z}) \varphi(z) \overline{n(z)}) \\
& +\frac{\overline{n(\varsigma)}}{4 r^{2}}((\varsigma-z)(\varsigma-z) \overline{\varphi(z)} n(z)+(\varsigma-z)(\bar{\varsigma}-\bar{z}) \varphi(z) n(z)), \\
& n_{i} n_{j} \varphi_{j}=n(\varsigma) \frac{1}{2}(n(z) \overline{\varphi(z)}+\overline{n(z)} \varphi(z)) \\
& =\frac{1}{4 r^{2}} n(\varsigma)(\varsigma-z)(\bar{\varsigma}-\bar{z}) 2(n(z) \overline{\varphi(z)}+\overline{n(z)} \varphi(z)) .
\end{aligned}
$$

Therefore

$$
\begin{aligned}
& H_{i, j}(x, y) \varphi_{j}(y)= \\
& =\frac{\mu}{4 \pi r^{4}} n(\varsigma)\{(\bar{\varsigma}-\bar{z})(\bar{\varsigma}-\bar{z}) \varphi(z) n(z)+(\varsigma-z)(\bar{\varsigma}-\bar{z}) \varphi(z) \overline{n(z)} \\
& +(\varsigma-z)(\bar{\varsigma}-\bar{z}) \overline{\varphi(z)} n(z)+(\varsigma-z)(\varsigma-z) \overline{\varphi(z) n(z)} \\
& -4(\varsigma-z)(\bar{\varsigma}-\bar{z}) \overline{\varphi(z)} n(z)-4(\varsigma-z)^{2} \overline{\varphi(z) n(z)} \\
& -4(\bar{\varsigma}-\bar{z})^{2} \varphi(z) n(z)-4(\bar{\varsigma}-\bar{z})(\varsigma-z) \varphi(z) \overline{n(z)} \\
& +(\varsigma-z)(\bar{\varsigma}-\bar{z}) \overline{\varphi(z)} n(z)+(\bar{\varsigma}-\bar{z})(\bar{\varsigma}-\bar{z}) \varphi(z) n(z) \\
& +(\varsigma-z)(\varsigma-z) \overline{\varphi(z) n(z)}+(\varsigma-z)(\bar{\varsigma}-\bar{z}) \varphi(z) \overline{n(z)} \\
& +2(\varsigma-z)(\bar{\varsigma}-\bar{z}) \overline{\varphi(z)} n(z)+2(\varsigma-z)(\bar{\varsigma}-\bar{z}) \varphi(z) \overline{n(z)}\} \\
& +\frac{\mu}{4 \pi r^{4}} \overline{n(\varsigma)}\{(\varsigma-z)(\bar{\varsigma}-\bar{z}) \varphi(z) n(z)+(\varsigma-z)(\varsigma-z) \varphi(z) \overline{n(z)} \\
& +(\varsigma-z)(\bar{\varsigma}-\bar{z}) \varphi(z) n(z)+(\varsigma-z)(\varsigma-z) \varphi(z) \overline{n(z)} \\
& -4(\varsigma-z)^{2} \overline{\varphi(z)} n(z)-4 \frac{(\varsigma-z)^{3}}{(\bar{\varsigma}-\bar{z})} \overline{\varphi(z) n(z)} \\
& -4(\varsigma-z)(\bar{\varsigma}-\bar{z}) \varphi(z) n(z)-4(\varsigma-z)^{2} \varphi(z) \overline{n(z)} \\
& +(\varsigma-z)(\varsigma-z) \overline{\varphi(z)} n(z)+(\varsigma-z)(\bar{\varsigma}-\bar{z}) \varphi(z) n(z) \\
& +(\varsigma-z)(\varsigma-z) \overline{\varphi(z)} n(z)+(\varsigma-z)(\bar{\varsigma}-\bar{z}) \varphi(z) n(z)\}
\end{aligned}
$$




$$
\begin{aligned}
& =\frac{-\mu}{2 \pi r^{4}}\left\{\left((\bar{\varsigma}-\bar{z})^{2} \varphi(z) n(z)+(\varsigma-z)^{2} \overline{\varphi(z) n(z)}\right) n(\varsigma)\right. \\
& \left.+\left((\varsigma-z)^{2}(\varphi(z) \overline{n(z)}+\overline{\varphi(z)} n(z))+2 \frac{(\varsigma-z)^{3}}{(\bar{\varsigma}-\bar{z})} \overline{\varphi(z) n(z)}\right) \overline{n(\varsigma)}\right\} \\
& =-\frac{\mu}{2 \pi}\left\{\left(\frac{1}{(\varsigma-z)^{2}} \varphi(z) n(z)+\frac{1}{(\bar{\varsigma}-\bar{z})^{2}} \overline{\varphi(z) n(z)}\right) n(\varsigma)\right. \\
& \left.+\left(\frac{1}{(\bar{\varsigma}-\bar{z})^{2}}(\varphi(z) \overline{n(z)}+\overline{\varphi(z)} n(z))+\frac{2(\varsigma-z)}{(\bar{\varsigma}-\bar{z})^{3}} \overline{\varphi(z) n(z)}\right) \overline{n(\varsigma)}\right\} .
\end{aligned}
$$

This finishes the proof of the theorem. $\square$

\subsection{Symmetric complex boundary element scheme}

We suppose that $\Gamma_{h}=\Gamma_{1 h} \cup \Gamma_{2 h}$ is quasi-uniform discretization of $\Gamma$ and

$$
\Gamma_{1 h}=\bigcup_{s=1}^{N_{1}}\left[z_{s}, z_{s+1}\right], \Gamma_{2 h}=\bigcup_{t=0}^{N_{2}-1}\left[z_{t}, z_{t+1}\right], N_{1}+N_{2}=: N
$$

where the order of nodes is put along the curve as counter-clockwise. Define boundary element spaces

$$
\begin{aligned}
S_{h} & :=S_{h}^{1} \times S_{h}^{2} \subset \widetilde{V}_{\Gamma}, \\
S_{h}^{1} & :=\operatorname{span}\left\{w_{s}: \operatorname{supp}\left(w_{s}\right) \subset \Gamma_{1 h}, s=1, \cdots, N_{1}\right\}, \\
S_{h}^{2} & :=\operatorname{span}\left\{\phi_{t}: \operatorname{supp}\left(\phi_{t}\right) \subset \Gamma_{2 h}, t=1, \cdots, N_{2}\right\} .
\end{aligned}
$$

Here basis functions are

$$
\begin{aligned}
& w_{s}(z)=\left\{\begin{array}{ll}
1 & z \in\left[z_{s}, z_{s+1}\right] \\
0 & z \notin\left[z_{s}, z_{s+1}\right]
\end{array},\right. \\
& \phi_{t}(z)=\left\{\begin{array}{ll}
\left(z_{t-1}-z\right) /\left(z_{t-1}-z_{t}\right) & z \in\left[z_{t-1}, z_{t}\right] \\
\left(z_{t+1}-z\right) /\left(z_{t+1}-z_{t}\right) & z \in\left[z_{t}, z_{t+1}\right] \\
0 & z \notin\left[z_{t-1}, z_{t}\right] \cup\left[z_{t}, z_{t+1}\right]
\end{array} .\right.
\end{aligned}
$$

Then, we get a discrete equation based on Galerkin method

$$
\begin{aligned}
& \left\langle\left[\begin{array}{cc}
V_{h} & -K_{h} \\
K_{h}^{\prime} & D_{h}
\end{array}\right]\left[\begin{array}{c}
\tau_{h}^{0} \\
\varphi_{h}^{0}
\end{array}\right],\left[\begin{array}{c}
\xi_{h} \\
p_{h}
\end{array}\right]\right\rangle= \\
& =\left\langle\left[\begin{array}{cc}
-V_{h} & 1 / 2 I+K_{h} \\
1 / 2 I-K_{h}^{\prime} & -D_{h}
\end{array}\right]\left[\begin{array}{c}
\hat{\tau} \\
\hat{\varphi}
\end{array}\right],\left[\begin{array}{c}
\xi_{h} \\
p_{h}
\end{array}\right]\right\rangle \quad \forall\left[\begin{array}{c}
\xi_{h} \\
p_{h}
\end{array}\right] \in S_{h},
\end{aligned}
$$


where $V_{h}, K_{h}, K_{h}^{\prime}, D_{h}$, which are discretizations of $V, K, K^{\prime}, D$ are defined on space $S_{h}$, and the unknown vector is $\left[\begin{array}{c}\tau_{h}^{0} \\ \varphi_{h}^{0}\end{array}\right] \in S_{h}^{0}:=\widetilde{V}_{\Gamma} \cap S_{h}$. Putting $\tau_{h}^{0}=\sum_{s=1}^{N_{1}} \alpha_{s} w_{s}$ and $\varphi_{h}^{0}=\sum_{t=1}^{N_{2}} \beta_{t} \phi_{t}$, we get a system of equations equivalent to (21)

$$
\begin{array}{ll}
\sum_{s=1}^{N_{1}}\left\langle V_{h} \alpha_{s} w_{s}, w_{s^{\prime}}\right\rangle-\sum_{t=1}^{N_{2}}\left\langle K_{h} \beta_{t} \phi_{t}, w_{s^{\prime}}\right\rangle=\left\langle\hat{b}, w_{s^{\prime}}\right\rangle \quad s^{\prime}=1, \cdots, N_{1}, \\
\sum_{s=1}^{N_{1}}\left\langle K_{h}^{\prime} \alpha_{s} w_{s}, \phi_{t^{\prime}}\right\rangle+\sum_{t=1}^{N_{2}}\left\langle D_{h} \beta_{t} \phi_{t}, \phi_{t^{\prime}}\right\rangle=\left\langle\hat{c}, \phi_{t^{\prime}}\right\rangle \quad t^{\prime}=1, \cdots, N_{2} .
\end{array}
$$

The potentials in the left hand side of (22) are calculated, respectively, by the potential expressions of (13)-(16) on $\Gamma_{h}$.

In real variable, $(22)$ are the system of $2 N$ order. We split the $2 N \times 2 N-$ coefficient matrix into four blocks by $V_{h}, K_{h}, K_{h}^{\prime}, D_{h}$. Then we get

Theorem 2.2 Assume that a hypersigular integral in the left hand side of (11) has a finite value. Then equation (11) has a unique solution $\left[\begin{array}{l}\tau^{0} \\ \varphi^{0}\end{array}\right] \in \widetilde{V}_{\Gamma}$.

Assume that total of hypersigular integrals of every element in $D_{h}$-blocks has finite value. Then, $V_{h}$ and $D_{h}$-blocks are, respectively, symmetric, $K_{h}$ and $K_{h}^{\prime}$-blocks are block skewsymmetric each other, and (21) has a unique solution $\left[\begin{array}{l}\tau_{h}^{0} \\ \varphi_{h}^{0}\end{array}\right] \in S_{h}^{0}$. Furthermore, if condition $\left[\begin{array}{l}\tau^{0} \\ \varphi^{0}\end{array}\right] \in \widetilde{H}^{-1 / 2+\sigma}\left(\Gamma_{1}\right) \times \widetilde{H}^{1 / 2+\sigma}\left(\Gamma_{2}\right), \sigma<\frac{1}{2}$ holds, then we have error estimation

$$
\begin{aligned}
& \left\|\left[\begin{array}{c}
\tau^{0} \\
\varphi^{0}
\end{array}\right]-\left[\begin{array}{l}
\tau_{h}^{0} \\
\varphi_{h}^{0}
\end{array}\right]\right\|_{H^{t-1}(\Gamma) \times H^{t}(\Gamma)} \leq C N^{t-s}\left\|\left[\begin{array}{c}
\tau^{0} \\
\varphi^{0}
\end{array}\right]\right\|_{H^{s-1}(\Gamma) \times H^{s}(\Gamma)}, \\
& -\frac{1}{2} \leq t \leq s \leq \sigma+\frac{1}{2} .
\end{aligned}
$$

Proof. The operator $V$ is a self-adjoint operator satisfying

$$
\langle V \tau, \tau\rangle_{L_{2}(\Gamma)} \geq c_{1}^{V\|\tau\|_{H^{-\frac{1}{2}}(\Gamma)}^{2}}, \forall \tau \in H^{-\frac{1}{2}}(\Gamma), \exists c_{1}^{V}>0,
$$

and the operator $D$ is a self-adjoint operator satisfying

$$
\langle D \varphi, \varphi\rangle_{L_{2}(\Gamma)} \geq c_{2}^{D}\|\varphi\|_{H^{\frac{1}{2}}(\Gamma)}^{2}, \forall \varphi \in H^{\frac{1}{2}}(\Gamma) / \operatorname{ker}(D), \quad \exists c_{2}^{D}>0 .
$$

In the case of linear elastic potential operators this result is already proved (Theorem 2 in [7]). Regarding the Stokes flow potential operator as special case of linear elastic potential operators with $\nu=\frac{1}{2}$, we get (24), (25).

Since

$$
-\langle K \varphi, \tau\rangle+\left\langle\varphi, K^{\prime} \tau\right\rangle=-\left\langle\varphi, K^{\prime} \tau\right\rangle+\left\langle\varphi, K^{\prime} \tau\right\rangle=0,
$$


we have

$$
a\left(\left[\begin{array}{l}
\tau \\
\varphi
\end{array}\right],\left[\begin{array}{l}
\tau \\
\varphi
\end{array}\right]\right) \geq c\left\|\left[\begin{array}{l}
\tau \\
\varphi
\end{array}\right]\right\|_{\widetilde{V}_{\Gamma}}^{2} \forall\left[\begin{array}{l}
\tau \\
\varphi
\end{array}\right] \in \widetilde{V}_{\Gamma}
$$

where $c=\min \left\{c_{1}^{V}, c_{2}^{D}\right\}$. By Lax-Milgram theorem we get unique existence of a solution to (11) and in the same way we get the solution for (21).

We can get the error estimation of the (23) extending the results of Theorem 1 in [17] for scalar to vector space. The symmetry of matrix-blocks will be described in detail in A2 of the the Appendix.

\section{Analytical calculation of hypersingular integrals}

In (22), integrals $\left\langle V_{h} \alpha_{s} w_{s}, w_{s^{\prime}}\right\rangle$ and $\left\langle K_{h} \beta_{t} \phi_{t}, w_{s^{\prime}}\right\rangle,\left\langle K_{h}^{\prime} \alpha_{s} w_{s}, \phi_{t^{\prime}}\right\rangle$ contain weak singular integrals, and $\left\langle D_{h} \beta_{t} \phi_{t}, \phi_{t^{\prime}}\right\rangle$ have hypersingular integrals.

\subsection{Calculation of hypersingular boundary integral by boundary limit method}

In this subsection we apply the boundary limit method in [19] to hypersingular boundary integrals given on the boundary $\Gamma$ of domain $\Omega$ in complex plane $\mathbb{C}$.

In the boundary element scheme in $\mathbb{R}^{d}(d=2,3)$, let us consider the definition of hypersingular boundary integral(cf. (1.2.6) in [11])

$$
D u(x)=-\lim _{x^{\prime} \rightarrow x \in \Gamma, x^{\prime} \in \Omega} \nabla_{x^{\prime}} \cdot n_{x} \int_{\Gamma} \frac{\partial E}{\partial n_{y}}\left(x^{\prime}, y\right) u(y) d \Gamma_{y} .
$$

To be clear, we assume a point $x^{\prime}$ be on the normal line passing point $x$. Since $x^{\prime} \in \Omega, y \in \Gamma$, it implies $\left|x^{\prime}-y\right|>0$, therefore

$$
\nabla_{x^{\prime}} \cdot n_{x} \int_{\Gamma} \frac{\partial E}{\partial n_{y}}\left(x^{\prime}, y\right) u(y) d \Gamma(y)=\int_{\Gamma} \frac{\partial^{2} E}{\partial n_{x} \partial n_{y}}\left(x^{\prime}, y\right) u(y) d \Gamma(y)
$$

because $\frac{\partial E}{\partial n_{y}}\left(x^{\prime}, y\right)$ is smooth and so differential symbol $\nabla_{x^{\prime}} \cdot n_{x}=\frac{\partial}{\partial n_{x}}$ can be entered into integral symbol. Putting $H\left(x^{\prime}, y\right):=-\frac{\partial^{2} E}{\partial n_{x} \partial n_{y}}\left(x^{\prime}, y\right)$, we get

$$
D u(x)=\lim _{x^{\prime} \rightarrow x \in \Gamma, x^{\prime} \in \Omega} \int_{\Gamma} H\left(x^{\prime}, y\right) u(y) d \Gamma(y) .
$$

Denoting $\left|x^{\prime}-x\right|=\varepsilon$, we have $x^{\prime}=x-n_{x} \varepsilon$, and

$$
D u(x)=\lim _{\varepsilon \rightarrow 0} \int_{\Gamma} H\left(x-n_{x} \varepsilon, y\right) u(y) d \Gamma_{y} .
$$


Formula (27) is boundary limit scheme in [19].

In the case of complex variable, definition (26) of hypersingular boundary integral can be written as follows

$$
D u(\varsigma)=\lim _{\Omega \ni \varsigma^{\prime} \rightarrow \varsigma \in \Gamma} \int_{\Gamma} H\left(\varsigma^{\prime}, z\right) u(z) d \Gamma(z) .
$$

For simplicity of notation, we used the same function symbols without change.

Assume a point $\varsigma^{\prime}$ is on the normal line passing the point $\varsigma$ and $n\left(\varsigma^{\prime}\right)=n(\varsigma)$. If the point $\varsigma^{\prime}$ be away $\varepsilon$ outside from the boundary, then $\varsigma^{\prime}=\varsigma+\varepsilon n(\varsigma)$ and

$$
D u(\varsigma)=\lim _{\varepsilon \rightarrow 0} \int_{\Gamma} H(\varsigma+\varepsilon n(\varsigma), z) u(z) d \Gamma(z) .
$$

Denoting by $t(\varsigma)$ the tangent vector with counter-clockwise along the boundary curve at the point $\varsigma^{\prime}$, we have $n(\varsigma)=-i t(\varsigma)$, and so (26) may written as follows.

$$
D u(\varsigma)=\lim _{\varepsilon \rightarrow 0} \int_{\Gamma} H(\varsigma-i \varepsilon t(\varsigma), z) u(z) d \Gamma(z) .
$$

As we see, boundary limit method requires only integrability of density $u(y)$. Therefore, when $u(z)$ is continue we can apply boundary limit method. Sign "-" before $i \varepsilon$ in (30) means approach process from outside to boundary. In contrast, sign "+" means from inside to boundary.

\subsection{Calculation of hypersingular matrix element}

As mentioned at the beginning of this section, hypersingular integrals appear in calculation of elements of block matrices $\left\langle D_{h} \beta_{t} \phi_{t}, \phi_{t^{\prime}}\right\rangle, t, t^{\prime}=1, \ldots, N_{2}$. In more detail, it is only when supports of basis functions $\phi_{t}$ and $\phi_{t^{\prime}}$ are overlapped, that is, $t^{\prime}=t$ (supports are coincided completely) or $\left|t^{\prime}-t\right|=1$ (supports are overlapped by half)

Let

$$
e_{t}:=z_{t+1}-z_{t}, L_{t}:=\left|e_{t}\right|, t=1, \ldots, N_{2}
$$

and

$$
\begin{aligned}
& z=z_{t}+\xi e_{t}, z \in\left[z_{t}, z_{t+1}\right], 0 \leq \xi \leq 1, \\
& \varsigma=z_{t}+\eta e_{t}, \varsigma \in\left[z_{t}, z_{t+1}\right], 0 \leq \eta \leq 1 .
\end{aligned}
$$

Then, we get

$$
\begin{aligned}
& \phi_{t}(z)=\left\{\begin{array}{ll}
\xi & z \in\left[z_{s-1}, z_{t}\right] \\
1-\xi, & z \in\left[z_{t}, z_{t+1}\right]
\end{array},\right. \\
& n(z)=-i e_{t} / L_{t}, \quad d \Gamma(z)=L_{t} d \xi, \quad z \in\left[z_{t}, z_{t+1}\right] .
\end{aligned}
$$




\subsubsection{Calculation of matrix element $\left\langle D_{h} \beta_{t} \phi_{t}, \phi_{t^{\prime}}\right\rangle, t=t^{\prime}$}

Since

$$
\operatorname{supp}\left(\phi_{t}(z)\right)=\operatorname{supp}\left(\phi_{t^{\prime}}(\varsigma)\right)=\left[z_{t-1}, z_{t}\right] \cup\left[z_{t}, z_{t+1}\right],
$$

interior integral variable $z$ and exterior integral variable $\varsigma$ of multiple integral $\int_{z_{t-1}}^{z_{t+1}} d \Gamma(\varsigma) \int_{z_{t-1}}^{z_{t+1}} d \Gamma(z)$ are met each other in integral process, and the integral is hypersingular. Using expression (16) of hypersingular potential, we have

$$
\begin{aligned}
& \left\langle D_{h} \beta_{t} \phi_{t}, \phi_{t}\right\rangle= \\
& -\frac{\mu}{2 \pi} \int_{z_{t-1}}^{z_{t+1}} \phi_{t}(\varsigma) d \Gamma(\varsigma) \int_{z_{t-1}}^{z_{t+1}}\left(\frac{n(z) \beta_{t} \phi_{t}(z)}{(\varsigma-z)^{2}}+\frac{\overline{n(z) \beta_{t} \phi_{t}(z)}}{(\bar{\varsigma}-\bar{z})^{2}}\right) n(\varsigma) \\
& \left.+\left(\frac{1}{(\bar{\varsigma}-\bar{z})^{2}}\left(n(z) \overline{\beta_{t} \phi_{t}(z)}+\overline{n(z)} \beta_{t} \phi_{t}(z)\right)+\frac{2(\varsigma-z)}{(\bar{\varsigma}-\bar{z})^{3}} \overline{n(z) \beta_{t} \phi_{t}(z)}\right) \overline{n(\varsigma)}\right) d \Gamma(z) \\
& =-\frac{\mu}{2 \pi} \beta_{t} \int_{z_{t+1}}^{z_{t-1}} \phi_{t}(\varsigma) d \Gamma(\varsigma) \int_{z_{t-1}}^{z_{t+1}}\left(\frac{1}{(\varsigma-z)^{2}} n(z) n(\varsigma)+\frac{1}{(\bar{\varsigma}-\bar{z})^{2}} \overline{n(z) n(\varsigma)}\right) \phi_{t}(z) d \Gamma(z) \\
& \left.-\frac{\mu}{2 \pi} \bar{\beta}_{t} \int_{z_{t-1}}^{z_{t+1}} \phi_{t}(\varsigma) d \Gamma(\varsigma) \int_{z_{t-1}}^{\left(\frac{n(z)}{n} n(\varsigma)+n(z) \overline{n(\varsigma)}\right.}+\frac{2(\varsigma-z)}{(\bar{\varsigma}-\bar{z})^{3}} \overline{n(z) n(\varsigma)}\right) \overline{\phi_{t}(z)} d \Gamma(z) .
\end{aligned}
$$

Splitting the last two integral terms into elements (suppressing $-\frac{\mu}{2 \pi}$ and integrands), we have

$$
\begin{aligned}
& \beta_{t} \int_{z_{t-1}}^{z_{t+1}}=\beta_{t} \int_{z_{t-1}}^{z_{t+1}} \int_{z_{t-1}}^{z_{t}}+\beta_{t} \int_{z_{t-1}}^{z_{t}} \int_{z_{t}}^{z_{t+1}}+\beta_{t} \int_{z_{t}}^{z_{t+1}} \int_{z_{t-1}}^{z_{t}}+\beta_{t} \int_{z_{t}}^{z_{t+1}} \int_{z_{t}}^{z_{t+1}} \\
& =: I_{1}\left(\beta_{t}\right)+I_{2}\left(\beta_{t}\right)+I_{3}\left(\beta_{t}\right)+I_{4}\left(\beta_{t}\right), \\
& \bar{\beta}_{t} \int_{z_{t-1}}^{z_{t+1}}=\bar{\beta}_{t} \int_{z_{t-1}}^{z_{t+1}}+\int_{z_{t-1}}^{z_{t}}+\bar{\beta}_{t} \int_{z_{t-1}}^{z_{t}}+\bar{\beta}_{t} \int_{z_{t}}^{z_{t+1}} \int_{z_{t-1}}^{z_{t}}+\bar{\beta}_{t} \int_{z_{t}}^{z_{t+1}} \int_{z_{t}}^{z_{t+1}} \\
& =: I_{1}\left(\bar{\beta}_{t}\right)+I_{2}\left(\bar{\beta}_{t}\right)+I_{3}\left(\bar{\beta}_{t}\right)+I_{4}\left(\bar{\beta}_{t}\right) .
\end{aligned}
$$

Finally, we obtain

$$
\left\langle D_{h} \beta_{t} \phi_{t}, \phi_{t}\right\rangle=-\frac{\mu}{2 \pi} I_{1}-\frac{\mu}{2 \pi} I_{2}-\frac{\mu}{2 \pi} I_{3}-\frac{\mu}{2 \pi} I_{4},
$$

where

$$
I_{k}=I_{k}\left(\beta_{t}\right)+I_{k}\left(\bar{\beta}_{t}\right), \quad k=1,2,3,4 .
$$


All these integrals are calculated in A4.1 of Appendix.

Among above integrals only $I_{1}\left(\beta_{1}\right), I_{2}\left(\beta_{2}\right), I_{3}\left(\beta_{3}\right), I_{4}\left(\beta_{4}\right)$ have, respectively, hypersingularity which imply appearance of divergent terms. But following two theorems shown this divergent terms cancel each other out.

First, from the computation of $I_{1}\left(\beta_{t}\right)$ and $I_{2}\left(\beta_{t}\right)$ we get following

Theorem 3.1 The first two integrals $-\frac{\mu}{2 \pi} I_{1}$ and $-\frac{\mu}{2 \pi} I_{2}$ in calculation of element $\left\langle D_{h} \beta_{t} \phi_{t}, \phi_{t}\right\rangle$ have, respectively, divergent terms $\frac{\mu}{2 \pi} \beta_{t} \log \left(\varepsilon^{2}\right)$ and $-\frac{\mu}{2 \pi} \beta_{t} \log \left(\varepsilon^{2}\right)$.

They are cancelled in the sum $-\frac{\mu}{2 \pi} I_{1}-\frac{\mu}{2 \pi} I_{2}$ and we get finite integral value

$$
\begin{aligned}
& -\frac{\mu}{2 \pi} I_{1}-\frac{\mu}{2 \pi} I_{2}=\beta_{t}\left(-\frac{\mu}{4 \pi}\right)\left(-\frac{e_{t-1}}{e_{t}} \log \left(e_{t-1}\right)-\left(2+\frac{e_{t}}{e_{t-1}}\right) \log \left(e_{t}\right)\right. \\
& +\left(2+\frac{e_{t-1}}{e_{t}}+\frac{e_{t}}{e_{t-1}}\right) \log \left(e_{t-1}+e_{t}\right)-\frac{\bar{e}_{t-1}}{\bar{e}_{t}} \log \left(\bar{e}_{t-1}\right)-\left(2+\frac{\bar{e}_{t}}{\bar{e}_{t-1}}\right) \log \left(\bar{e}_{t}\right) \\
& \left.+\left(2+\frac{\bar{e}_{t-1}}{\bar{e}_{t}}+\frac{\bar{e}_{t}}{\bar{e}_{t-1}}\right) \log \left(\bar{e}_{t-1}+\bar{e}_{t}\right)\right) \\
& +\bar{\beta}_{t}\left(-\frac{\mu}{4 \pi}\right)\left(3 \frac{e_{t-1}}{\bar{e}_{t-1}}+\frac{e_{t}}{\bar{e}_{t}}-\left(\frac{e_{t} \bar{e}_{t-1}}{\bar{e}_{t}^{2}}-\frac{e_{t-1}}{\bar{e}_{t}}\right) \log \left(\bar{e}_{t-1}\right)\right. \\
& \left.+\left(\frac{e_{t}}{\bar{e}_{t-1}}-\frac{e_{t-1} \bar{e}_{t}}{\bar{e}_{t-1}^{2}}\right) \log \left(\bar{e}_{t}\right)\left(\frac{e_{t} \bar{e}_{t-1}}{\bar{e}_{t}^{2}}-\frac{e_{t-1}}{\bar{e}_{t}}-\frac{e_{t}}{\bar{e}_{t-1}}+\frac{e_{t-1} \bar{e}_{t}}{\bar{e}_{t-1}^{2}}\right) \log \left(\bar{e}_{t-1}+\bar{e}_{t}\right)\right) .
\end{aligned}
$$

Also from the computation of $I_{3}\left(\beta_{t}\right)$ and $I_{4}\left(\beta_{t}\right)$ we get following

Theorem 3.2 Last two integrals $-\frac{\mu}{2 \pi} I_{3}$ and $-\frac{\mu}{2 \pi} I_{4}$ of (38) in calculation of hypersingular element $\left\langle D_{h} \beta_{t} \phi_{t}, \phi_{t}\right\rangle$ have, respectively, divergent terms $-\frac{\mu}{2 \pi} \beta_{t} \log \left(\varepsilon^{2}\right)$ and $\frac{\mu}{2 \pi} \beta_{t} \log \left(\varepsilon^{2}\right)$. These are cancelled in sum $-\frac{\mu}{2 \pi} I_{3}-\frac{\mu}{2 \pi} I_{4}$ and we get the following finite integral value.

$$
\begin{aligned}
& -\frac{\mu}{2 \pi} I_{3}-\frac{\mu}{2 \pi} I_{4}=\beta_{t}\left(-\frac{\mu}{4 \pi}\right) \\
& \times\left(-\frac{e_{t}}{e_{t-1}} \log \left(e_{t}\right)-\left(2+\frac{e_{t-1}}{e_{t}}\right) \log \left(e_{t-1}\right)+\left(2+\frac{e_{t-1}}{e_{t}}+\frac{e_{t}}{e_{t-1}}\right) \log \left(e_{t}+e_{t-1}\right)\right. \\
& \left.-\frac{\bar{e}_{t}}{\bar{e}_{t-1}} \log \left(\bar{e}_{t}\right)-\left(2+\frac{\bar{e}_{t-1}}{\bar{e}_{t}}\right) \log \left(\bar{e}_{t-1}\right)+\left(2+\frac{\bar{e}_{t-1}}{\bar{e}_{t}}+\frac{\bar{e}_{t}}{\bar{e}_{t-1}}\right) \log \left(\bar{e}_{t}+\bar{e}_{t-1}\right)\right) \\
& +\bar{\beta}_{t}\left(-\frac{\mu}{4 \pi}\right)\left(3 \frac{e_{t}}{\bar{e}_{t}}+\frac{e_{t-1}}{\bar{e}_{t-1}}-\left(\frac{e_{t-1} \bar{e}_{t}}{\bar{e}_{t-1}^{2}}-\frac{e_{t}}{\bar{e}_{t-1}}\right) \log \left(\bar{e}_{t}\right)+\left(\frac{e_{t-1}}{\bar{e}_{t}}-\frac{e_{t} \bar{e}_{t-1}}{\bar{e}_{t}^{2}}\right)\right. \\
& \left.\times \log \left(\bar{e}_{t-1}\right)+\left(\frac{e_{t-1} \bar{e}_{t}}{\bar{e}_{t-1}^{2}}-\frac{e_{t}}{\bar{e}_{t-1}}-\frac{e_{t-1}}{\bar{e}_{t}}+\frac{e_{t} \bar{e}_{t-1}}{\bar{e}_{t}^{2}}\right) \log \left(\bar{e}_{t}+\bar{e}_{t-1}\right)\right) .
\end{aligned}
$$


From all integrals of (39), which are calculated in A4.1, we have

Theorem 3.3 Divergent terms being canceled, hypersingular elements $\left\langle D_{h} \beta_{t} \phi_{t}\right.$, $\left.\phi_{t}\right\rangle, t=1, \ldots, N_{2}$, have the following finite integral value.

$$
\begin{aligned}
& \left\langle D_{h} \beta_{t} \phi_{t}, \phi_{t}\right\rangle= \\
& =\beta_{t}\left(-\frac{\mu}{2 \pi}\right)\left(-\left(1+\frac{e_{t-1}}{e_{t}}\right) \log \left(e_{t-1}\right)-\left(1+\frac{e_{t}}{e_{t-1}}\right) \log \left(e_{t}\right)\right. \\
& +\left(2+\frac{e_{t-1}}{e_{t}}+\frac{e_{t}}{e_{t-1}}\right) \log \left(e_{t-1}+e_{t}\right)-\left(1+\frac{\bar{e}_{t-1}}{\bar{e}_{t}}\right) \log \left(\bar{e}_{t-1}\right) \\
& \left.-\left(1+\frac{\bar{e}_{t}}{\bar{e}_{t-1}}\right) \log \left(\bar{e}_{t}\right)+\left(2+\frac{\bar{e}_{t-1}}{\bar{e}_{t}}+\frac{\bar{e}_{t}}{\bar{e}_{t-1}}\right) \log \left(\bar{e}_{t-1}+\bar{e}_{t}\right)\right) \\
& +\bar{\beta}_{t}\left(-\frac{\mu}{2 \pi}\right)\left(2\left(\frac{e_{t-1}}{\bar{e}_{t-1}}+\frac{e_{t}}{\bar{e}_{t}}\right)+\left(\frac{e_{t-1}}{\bar{e}_{t}}-\frac{e_{t} \bar{e}_{t-1}}{\bar{e}_{t}^{2}}\right) \log \left(\bar{e}_{t-1}\right)\right. \\
& \left.+\left(\frac{e_{t}}{\bar{e}_{t-1}}-\frac{e_{t-1} \bar{e}_{t}}{\bar{e}_{t-1}^{2}}\right) \log \left(\bar{e}_{t}\right)+\left(\frac{e_{t} \bar{e}_{t-1}}{\bar{e}_{t}^{2}}-\frac{e_{t-1}}{\bar{e}_{t}}-\frac{e_{t}}{\bar{e}_{t-1}}+\frac{e_{t-1} \bar{e}_{t}}{\bar{e}_{t-1}^{2}}\right) \log \left(\bar{e}_{t-1}+\bar{e}_{t}\right)\right)
\end{aligned}
$$

3.2.2 Calculation of matrix element $\left\langle D_{h} \beta_{t} \phi_{t}, \phi_{t^{\prime}}\right\rangle,\left|t-t^{\prime}\right|=1$

Without loss of generality, suppose that $t^{\prime}=t+1$. Then

$$
\operatorname{supp}\left(\phi_{t}(z)\right) \cap \operatorname{supp}\left(\phi_{t^{\prime}}(\varsigma)\right)=\left[z_{t}, z_{t+1}\right] .
$$

By (16) we get

$$
\begin{aligned}
& \left\langle D_{h} \beta_{t} \phi_{t}, \phi_{t^{\prime}}\right\rangle=\left\langle D_{h} \beta_{t} \phi_{t}, \phi_{t+1}\right\rangle= \\
& =-\frac{\mu}{2 \pi} \beta_{t} \int_{z_{t}}^{z_{t+2}} \phi_{t+1}(\varsigma) d \Gamma(\varsigma) \int_{z_{t-1}}^{z_{t+1}}\left(\frac{n(z) n(\varsigma)}{(\varsigma-z)^{2}}+\frac{\overline{n(z) n(\varsigma)}}{(\bar{\varsigma}-\bar{z})^{2}}\right) \phi_{t}(z) d \Gamma(z) \\
& -\frac{\mu}{2 \pi} \bar{\beta}_{t} \int_{z_{t}}^{z_{t+2}} \phi_{t+1}(\varsigma) d \Gamma(\varsigma) \int_{z_{t-1}}^{z_{t+1}}\left(\frac{\overline{n(z)} n(\varsigma)+n(z) \overline{n(\varsigma)}}{(\bar{\varsigma}-\bar{z})^{2}}+\frac{2(\varsigma-z)}{(\bar{\varsigma}-\bar{z})^{3}} \overline{n(z) n(\varsigma)}\right) \times \\
& \times \overline{\phi_{t}(z)} d \Gamma(z) .
\end{aligned}
$$

As (36) and (37) let us express two terms in the right hand side above formula 
as follows.

$$
\begin{array}{r}
\beta_{t} \int_{z_{t}}^{z_{t+2}}=\beta_{t} \int_{z_{t-1}}^{z_{t+1}} \int_{z_{t-1}}^{z_{t+1}}+\beta_{t} \int_{z_{t}}^{z_{t}} \int_{z_{t}}^{z_{t+1}}+\beta_{t} \int_{z_{t+1}}^{z_{t+1}}+\beta_{z_{t-1}}^{z_{t+2}} \int_{z_{t+1}}^{z_{t}} \int_{z_{t}}^{z_{t+2}} \\
=: I_{1}\left(\beta_{t}\right)+I_{2}\left(\beta_{t}\right)+I_{3}\left(\beta_{t}\right)+I_{4}\left(\beta_{t}\right), \\
\bar{\beta}_{t} \int_{z_{t}}^{z_{t+2}}=\bar{\beta}_{t} \int_{z_{t-1}}^{z_{t+1}} \int_{z_{t-1}}^{z_{t+1}}+\bar{\beta}_{t} \int_{z_{t}}^{z_{t+1}} \int_{z_{t}}^{z_{t+1}}+\bar{\beta}_{t} \int_{z_{t+1}}^{z_{t+2}} \int_{z_{t-1}}^{z_{t}}+\bar{\beta}_{t} \int_{z_{t+1}}^{z_{t+2}} \int_{z_{t}}^{z_{t+1}} \\
=: I_{1}\left(\bar{\beta}_{t}\right)+I_{2}\left(\bar{\beta}_{t}\right)+I_{3}\left(\bar{\beta}_{t}\right)+I_{4}\left(\bar{\beta}_{t}\right) .
\end{array}
$$

Among above integrals only $I_{2}\left(\beta_{2}\right)$ has divergent terms, but this divergent terms cancel each other out(Appendix:A4.2.), so we get

Theorem 3.4 For every pair $\left(t, t^{\prime}\right)$ such that $\left|t-t^{\prime}\right|=1$, element $\left\langle D_{h} \beta_{t} \phi_{t}, \phi_{t^{\prime}}\right\rangle$, canceling divergent terms each other, has a finite integral value.

As shown above, elements of boundary element matrix be calculated by various types of integrals, where the element corresponding to hypersingular integral can have divergent terms. If there exist a element that it's divergent terms are not cancelled, the scheme is useless.

From this point of view we define the compatibility of the scheme.

Definition 3.1 When for every element determined by hypersingular integral among elements of discrete matrices obtained in boundary element scheme, all divergent terms are cancelled each other, we say the scheme have compatibility.

Theorem 3.5 Galerkin scheme (22) has compatibility.

Proof. In the matrix calculation of the left hand side in (22) divergent terms are from $D_{h}$-block. In detail, divergent terms are from the hypersingular integral element $\left\langle D_{h} \beta_{t} \phi_{t}, \phi_{t^{\prime}}\right\rangle, t^{\prime}=t$ in which supports of basis functions in interior and exterior integral are coincided completely and the hypersingular integral element $\left\langle D_{h} \beta_{t} \phi_{t}, \phi_{t^{\prime}}\right\rangle,\left|t^{\prime}-t\right|=1$ in which the supports are coincided by half, and not from others in $D_{h}$-block.

Theorem 3.3 shows that divergent terms from hypersingular integral $\left\langle D_{h} \beta_{t} \phi_{t}, \phi_{t^{\prime}}\right\rangle, t^{\prime}=t$, are cancelled and Theorem 3.4 does the same for the case of hypersingular integral $\left\langle D_{h} \beta_{t} \phi_{t}, \phi_{t^{\prime}}\right\rangle,\left|t^{\prime}-t\right|=1$. Since all divergent terms are cancelled, the scheme has compatibility.

\section{Algorithm}

\section{Step 1. Construction of complex discrete equation}

In the matrix calculation of the final discrete equation (22) calculation of $V_{h}$ - block elements can be fulfilled by simple analytical calculation because operator $V_{h}$ is weak singular and basis functions $w_{s}, w_{s^{\prime}}$ are constants. 
For elements of $K_{h}, K_{h^{\prime}}$-blocks, supports of corresponding basis functions $\phi_{t}, w_{s^{\prime}}$ and $w_{s}, \phi_{t^{\prime}}$ have no subsection, which allow us to calculate them by quadrature.

For elements of $D_{h}$ - blocks, since in the case of $\left|t-t^{\prime}\right|>1$ supports of basis functions $\phi_{t}, \phi_{t^{\prime}}$ have no subsection or only one point as subsection, it can be calculated by quadrature or simple analytical integral.

Right hand side of discrete equation (22) may be written as follows.

$$
\begin{aligned}
& \left\langle\widehat{b}, w_{s^{\prime}}\right\rangle:=-\left\langle V_{h} \widehat{\tau}, w_{s^{\prime}}\right\rangle+\frac{1}{2}\left\langle\widehat{\varphi}, w_{s^{\prime}}\right\rangle+\left\langle K_{h} \widehat{\varphi}, w_{s^{\prime}}\right\rangle, \\
& \left\langle\widehat{c}, \phi_{t^{\prime}}\right\rangle:=\frac{1}{2}\left\langle\widehat{\tau}, \phi_{t^{\prime}}\right\rangle-\left\langle K_{h}^{\prime} \widehat{\tau}, \phi_{t^{\prime}}\right\rangle-\left\langle D_{h} \widehat{\varphi}, \phi_{t^{\prime}}\right\rangle
\end{aligned}
$$

where $\left[\begin{array}{l}\widehat{\tau} \\ \widehat{\varphi}\end{array}\right] \in V_{\Gamma}$ is arbitrarily taken with condition $\left.\widehat{\tau}\right|_{\Gamma_{2}}=g,\left.\widehat{\varphi}\right|_{\Gamma_{1}}=f$.

We take an approximation $\left[\begin{array}{c}\widehat{\tau}_{h} \\ \widehat{\varphi}_{h}\end{array}\right] \in V_{h}$ as follows.

$$
\widehat{\tau}_{h}=\sum_{s=1}^{N_{1}} \tau_{s} w_{s}+\sum_{t=1}^{N_{2}} \tau_{N_{1}+t} \phi_{t}, \widehat{\varphi}_{h}=\sum_{s=1}^{N_{1}} \varphi_{s} w_{s}+\sum_{t=1}^{N_{2}} \varphi_{N_{1}+t} \phi_{t}
$$

where $\tau_{s}, \varphi_{s}, 1 \leq s \leq N_{1}$ - element mean, $\tau_{N_{1}+t}, \varphi_{N_{1}+t} 1 \leq t \leq N_{2}$ - node value. Then, we get

$$
\begin{aligned}
&\left\langle V_{h} \widehat{\tau}, w_{s^{\prime}}\right\rangle=\sum_{s=1}^{N_{1}}\left\langle V_{h} \tau_{s} w_{s}, w_{s^{\prime}}\right\rangle+\sum_{t=1}^{N_{2}}\left\langle V_{h} \tau_{N_{1}+t} \phi_{t}, w_{s^{\prime}}\right\rangle \\
&\left\langle K_{h} \widehat{\varphi}, w_{s^{\prime}}\right\rangle=\sum_{s=1}^{N_{1}}\left\langle K_{h} \varphi_{s} w_{s}, w_{s^{\prime}}\right\rangle+\sum_{t=1}^{N_{2}}\left\langle K_{h} \varphi_{N_{1}+1} \phi_{t}, w_{s^{\prime}}\right\rangle \\
&\left\langle K_{h}^{\prime} \widehat{\tau}, \phi_{t^{\prime}}\right\rangle=\sum_{s=1}^{N_{1}}\left\langle K_{h}^{\prime} \tau_{s} w_{s}, \phi_{t^{\prime}}\right\rangle+\sum_{t=1}^{N_{2}}\left\langle K_{h}^{\prime} \tau_{N_{1}+t} \phi_{t}, \phi_{t^{\prime}}\right\rangle \\
&\left\langle D_{h} \widehat{\varphi}, \phi_{t^{\prime}}\right\rangle=\sum_{s=1}^{N_{1}}\left\langle D_{h} \varphi_{s} w_{s}, \phi_{t^{\prime}}\right\rangle+\sum_{t=1}^{N_{2}}\left\langle D_{h} \varphi_{N_{1}+1} \phi_{t}, \phi_{t^{\prime}}\right\rangle \\
&\left\langle\widehat{\varphi}, w_{s^{\prime}}\right\rangle=\varphi_{s^{\prime}},\left\langle\widehat{\tau}, \phi_{t^{\prime}}\right\rangle=\sum_{t=t^{\prime}-2}^{t^{\prime}+2} \tau_{N_{1}+t}\left\langle\phi_{t}, \phi_{t^{\prime}}\right\rangle
\end{aligned}
$$

Since the coefficients $\tau_{s}, \tau_{N_{1}+t}, \varphi_{s}, \varphi_{N_{1}+1}$ are known, the integrals above can be calculated as before.

Step 2. calculation of real discrete equation

Calculating every block of left hand side in (22), we get a complex discrete equation for $s=1, \ldots, N_{1}$ and $t=1, \ldots, N_{2}$

$$
\left[\begin{array}{ll}
A_{s} & B_{t} \\
C_{s} & D_{t}
\end{array}\right]\left[\begin{array}{c}
\alpha_{s} \\
\beta_{t}
\end{array}\right]+\left[\begin{array}{ll}
E_{s} & F_{t} \\
G_{s} & H_{t}
\end{array}\right]\left[\begin{array}{l}
\overline{\alpha_{s}} \\
\beta_{t}
\end{array}\right]=\left[\begin{array}{c}
R_{s, t} \\
S_{s, t}
\end{array}\right]
$$

Denoting complex matrices and vectors by $A_{s}=A_{s}^{(1)}+A_{s}^{(2)} i$ and $\alpha_{s}=\alpha_{s}^{(1)}+i \alpha_{s}^{(2)}$, 
we have the following real discrete equation

$$
\begin{aligned}
& {\left[\begin{array}{cccc}
A_{s}^{(1)}+E_{s}^{(1)} & -A_{s}^{(2)}+E_{s}^{(2)} & B_{t}^{(1)}+F_{t}^{(1)} & -B_{t}^{(2)}+F_{t}^{(2)} \\
C_{s}^{(1)}+G_{s}^{(1)} & -C_{s}^{(2)}+G_{s}^{(2)} & D_{t}^{(1)}+H_{t}^{(1)} & -D_{t}^{(2)}+H_{t}^{(2)} \\
A_{s}^{(2)}+E_{s}^{(2)} & A_{s}^{(1)}-E_{s}^{(1)} & B_{t}^{(2)}+F_{t}^{(2)} & B_{t}^{(1)}-F_{t}^{(1)} \\
C_{s}^{(2)}+G_{s}^{(2)} & C_{s}^{(1)}-G_{s}^{(1)} & D_{t}^{(2)}+H_{t}^{(2)} & D_{t}^{(1)}-H_{t}^{(1)}
\end{array}\right]\left[\begin{array}{c}
\alpha_{s}^{(1)} \\
\alpha_{s}^{(2)} \\
\beta_{t}^{(1)} \\
\beta_{t}^{(2)}
\end{array}\right]=} \\
& =\left[\begin{array}{llll}
R_{s, t}^{(1)} & S_{s, t}^{(1)} & R_{s, t}^{(2)} & S_{s, t}^{(2)}
\end{array}\right]^{T} .
\end{aligned}
$$

Denoting again the total unknown vector by

$$
\begin{aligned}
& \left(\alpha_{1}^{(1)}, \alpha_{1}^{(2)}, \ldots, \alpha_{N_{1}}^{(1)}, \alpha_{N_{1}}^{(2)}, \beta_{1}^{(1)}, \beta_{1}^{(2)}, \ldots, \beta_{N_{2}}^{(1)}, \beta_{N_{2}}^{(2)}\right)^{T} \\
& =\left(\alpha_{1}, \alpha_{2}, \ldots, \alpha_{2 N_{1}}, \alpha_{2 N_{1}+1}, \ldots, \alpha_{2 N}\right)^{T}=: X
\end{aligned}
$$

assembling to this vector we combine the total coefficient matrix as following

$$
\begin{gathered}
A=\left[\begin{array}{cccccc}
a_{1,1} & \ldots & a_{1,2 N_{1}} & a_{1,2 N_{1}+1} & \ldots & a_{1,2 N} \\
\ldots & \ldots & \ldots & \ldots & \ldots & \ldots \\
a_{2 N_{1}, 1} & \ldots & a_{2 N_{1}, 2 N_{1}} & a_{2 N_{1}, 2 N_{1}+1} & \ldots & a_{2 N_{1}, 2 N} \\
a_{2 N_{1}+1,1} & \ldots & a_{2 N_{1}+1,2 N_{1}} & a_{2 N_{1}+1,2 N_{1}+1} & \ldots & a_{2 N_{1}+1,2 N} \\
\ldots & \ldots & \ldots & \ldots & \ldots & \ldots \\
a_{2 N, 1} & \ldots & a_{2 N, 2 N_{1}} & a_{2 N, 2 N_{1}+1} & \ldots & a_{2 N, 2 N}
\end{array}\right] \\
=\left[\begin{array}{cc}
A_{V_{h}} & A_{K_{h}} \\
A_{K_{h}^{\prime}} & A_{D_{h}}
\end{array}\right]
\end{gathered}
$$

and denote the right hand side by $B$. Then We get the resulting equation

$$
A X=B
$$

Notes that In matrix $A$ block $A_{V_{h}}$ and block $A_{D_{h}}$ are symmetric,respectively, $A_{K_{h}}$ and $A_{K_{h}^{\prime}}$ is skewsymmetric.

By solving this equation, we can directly have the traction and the velocity on the boundary, that is,

$$
\begin{aligned}
& \left(\alpha_{s}^{(1)}+\tau_{s}^{(1)}, \alpha_{s}^{(2)}+\tau_{s}^{(2)}\right)-\text { traction for element }\left[z_{s}, z_{s+1}\right] \subset \Gamma_{1 h}, \\
& \left(\beta_{t}^{(1)}+\varphi_{N_{1}+t}^{(1)}, \beta_{t}^{(2)}+\varphi_{N_{1}+t}^{(2)}\right)-\text { velocity at node } z_{t} \in \Gamma_{2 h} .
\end{aligned}
$$

Step 3. Calculation of velocity and pressure in domain

To get the velocity and pressure at the required position in domain, we can use the formula

$$
\begin{aligned}
& u(\varsigma)=V_{h}(\widehat{\tau}+\widehat{\alpha})(\varsigma)-K_{h}(\widehat{\varphi}+\widehat{\beta})(\varsigma), \\
& p(\varsigma)=Q_{h}(\widehat{\tau}+\widehat{\alpha})(\varsigma)+R_{h}(\widehat{\varphi}+\widehat{\beta})(\varsigma), \quad \varsigma \in \Omega,
\end{aligned}
$$


where

$$
\begin{aligned}
& Q_{h} \tau(\varsigma)=\frac{1}{4 \pi} \int_{\Gamma}\left(\frac{1}{(\varsigma-z)} \tau(z)+\frac{1}{\bar{\varsigma}-\bar{z}} \overline{\tau(z)}\right) d \Gamma(z), \\
& R_{h} \varphi(\varsigma)=-\frac{\mu}{2 \pi} \int_{\Lambda}\left(\frac{1}{(\varsigma-z)^{2}} n(z) \varphi(z)+\frac{1}{(\bar{\varsigma}-\bar{z})^{2}} \overline{n(z) \varphi(z)}\right) d \Gamma(z), \\
& \widehat{\alpha}_{s}=\left\{\begin{array}{ll}
\alpha_{s}, & 1 \leq s \leq N_{1}, \\
0, & N_{1}<s \leq N,
\end{array} \widehat{\beta}_{t}= \begin{cases}0, & 1 \leq t \leq N_{1}, \\
\beta_{t-N_{1}}, & N_{1}<t \leq N .\end{cases} \right.
\end{aligned}
$$

\section{Conclusion}

In the paper, we realize symmetric complex boundary element scheme of 2-D Stokes mixed boundary value problem and proved the convergence, stability of the scheme. In particular, we presented the method to convert boundary element scheme into complex boundary element scheme directly. We got the analytical calculation formulae for hypersingular boundary integral in the scheme by boundary limit method, to do this, we proved cancelation properties of the divergent terms in hypersingular integrals.

\section{Appendix}

\section{A1. Proof of (i)-(v) in Subsection 2.1}

$$
\text { (i) } \begin{aligned}
& : r_{, j}(x, y) u_{j}(y)=-\frac{1}{r}\left(\left(x_{1}-y_{1}\right) u_{1}(y)+\left(x_{2}-y_{2}\right) u_{2}(y)\right) \\
& =-\frac{1}{r}\left(\frac{1}{2}(\varsigma-z+(\bar{\varsigma}-\bar{z})) u_{1}(y)+\frac{1}{2 i}(\varsigma-z-(\bar{\varsigma}-\bar{z})) u_{2}(y)\right) \\
& =-\frac{1}{2 r}\left((\varsigma-z)\left(u_{1}(y)-i u_{2}(y)\right)+(\bar{\varsigma}-\bar{z})\left(u_{1}(y)+i u_{2}(y)\right)\right) \\
& =-\frac{1}{2 r}((\varsigma-z) \overline{u(z)}+(\bar{\varsigma}-\bar{z}) u(z)) .
\end{aligned}
$$

(ii) : $n_{j}(y) u_{j}(y)=n_{1}(y) u_{1}(y)+n_{2}(y) u_{2}(y)$

$$
\begin{aligned}
& =\frac{1}{2}(n(z)+\overline{n(z)}) u_{1}(y)+\frac{1}{2 i}(n(z)-\overline{n(z)}) u_{2}(y) \\
& =\frac{1}{2}\left(n(z)\left(u_{1}(y)-i u_{2}(y)\right)+\overline{n(z)}\left(u_{1}(y)+i u_{2}(y)\right)\right) \\
& =\frac{1}{2}(n(z) \overline{u(z)}+\overline{n(z)} u(z)) .
\end{aligned}
$$

(iii), (iv) are proved similarly to (i). 


$$
\begin{aligned}
(\mathrm{v}) & : r_{, i}(x, y) r_{, j}(x, y) u_{j}(y)= \\
& =\frac{-1}{r}(\varsigma-z) \frac{-1}{2 r}((\varsigma-z) \overline{u(z)}+(\bar{\varsigma}-\bar{z}) u(z)) \\
& =\frac{\varsigma-z}{2 r^{2}}((\varsigma-z) \overline{u(z)}+(\bar{\varsigma}-\bar{z}) u(z)) \\
& =\frac{1}{2(\bar{\varsigma}-\bar{z})}((\varsigma-z) \overline{u(z)}+(\bar{\varsigma}-\bar{z}) u(z)) \\
& =\frac{1}{2}\left(\frac{\varsigma-z}{\bar{\varsigma}-\bar{z}} \overline{u(z)}+u(z)\right) .
\end{aligned}
$$

\section{A2. Proof of Symmetry of the Real Coefficient Matrix of the System} in $(22)$

Denote supports of basis in $\Gamma_{1 h}$ by $\pi_{1}, \ldots, \pi_{s}, \ldots, \pi_{N_{1}}$ and in $\Gamma_{2 h}$ by $\bar{\pi}_{1}, \ldots, \bar{\pi}_{t}, \ldots, \bar{\pi}_{N_{2}}$, respectively. Note that $\pi_{s}$ is element, $\bar{\pi}_{t}$ is sum of two elements which has common point $z_{t}$.

Subscripts $i, j$ of entries of the coefficient matrix are define as following.

$$
\begin{aligned}
& i=\left\{\begin{array}{ll}
2 s^{\prime}, & \pi=\pi_{s^{\prime}} \in \Gamma_{1 h}, s^{\prime}=1, \ldots, N_{1} \\
2 N_{1}+2 t^{\prime} & \pi=\bar{\pi}_{t^{\prime}} \in \Gamma_{2 h}, t^{\prime}=1, \ldots, N_{2}
\end{array},\right. \\
& j=\left\{\begin{array}{ll}
2 s, & \pi=\pi_{s} \in \Gamma_{1 h}, s=1, \ldots, N_{1} \\
2 N_{1}+2 t & \pi=\bar{\pi}_{t} \in \Gamma_{2 h}, t=1, \ldots, N_{2}
\end{array} .\right.
\end{aligned}
$$

Note that $i, j=1, \ldots, 2 N_{1}, 2 N_{1}+1, \ldots, 2 N$.

First, we will establish symmetry of $V_{h}$.-block

$$
\begin{aligned}
& \left\langle V_{h} \alpha_{s} w_{s}, w_{s^{\prime}}\right\rangle=\frac{1}{8 \pi \mu} \int_{\Gamma} w_{s^{\prime}}(\varsigma) d \Gamma(\varsigma) \times \\
& \times \int_{\Gamma}\left(\log \frac{1}{\varsigma-z} \alpha_{s} w_{s}(z)+\log \frac{1}{\bar{\varsigma}-\bar{z}} \alpha_{s} w_{s}(z)+\frac{\varsigma-z}{\bar{\varsigma}-\bar{z}} \overline{\alpha_{s} w_{s}(z)}\right) d \Gamma(z) \\
& =\frac{1}{8 \pi \mu} \int_{\pi_{s^{\prime}}} d \Gamma(\varsigma) \int_{\pi_{s}}\left(\log \frac{1}{\varsigma-z} \alpha_{s}+\log \frac{1}{\bar{\varsigma}-\bar{z}} \alpha_{s}+\frac{\varsigma-z}{\bar{\varsigma}-\bar{z}} \overline{\alpha_{s}}\right) d \Gamma(z)
\end{aligned}
$$


By Theorem 2.1, in real variable,

$$
\begin{aligned}
& \left\langle V_{h} \alpha_{s} w_{s}, w_{s^{\prime}}\right\rangle=\frac{1}{4 \pi \mu} \int_{\pi_{s^{\prime}}} d \Gamma(x) \int_{\pi_{s}}\left(\left(\log \frac{1}{|x-y|}-\frac{1}{2}\right) I\right. \\
& \left.+\frac{1}{|x-y|^{2}}\left[\begin{array}{cc}
\left(x_{1}-y_{1}\right)^{2} & \left(x_{1}-y_{1}\right)\left(x_{2}-y_{2}\right) \\
\left(x_{2}-y_{2}\right)\left(x_{1}-y_{1}\right) & \left(x_{2}-y_{2}\right)^{2}
\end{array}\right]\right) d \Gamma(y)\left[\begin{array}{c}
\alpha_{s}^{(1)} \\
\alpha_{s}^{(2)}
\end{array}\right] \\
& =:\left[\begin{array}{ll}
a_{11 ; s^{\prime} s} & a_{12 ; s^{\prime} s} \\
a_{21 ; s^{\prime} s} & a_{22 ; s^{\prime} s}
\end{array}\right]\left[\begin{array}{l}
\alpha_{s}^{(1)} \\
\alpha_{s}^{(2)}
\end{array}\right] ; \\
& a_{11 ; s^{\prime} s}=\frac{1}{4 \pi \mu} \int_{\pi_{s^{\prime}}} d \Gamma(x) \int_{\pi_{s}}\left(\log \frac{1}{|x-y|}-\frac{1}{2}+\frac{\left(x_{1}-y_{1}\right)^{2}}{|x-y|^{2}}\right) d \Gamma(y) \\
& a_{12 ; s^{\prime} s}=\frac{1}{4 \pi \mu} \int_{\pi_{s^{\prime}}} d \Gamma(x) \int_{\pi_{s}} \frac{\left(x_{1}-y_{1}\right)\left(x_{2}-y_{2}\right)}{|x-y|^{2}} d \Gamma(y), \\
& a_{21 ; s^{\prime} s}=\frac{1}{4 \pi \mu} \int_{\pi_{s^{\prime}}} d \Gamma(x) \int_{\pi_{s}} \frac{\left(x_{2}-y_{2}\right)\left(x_{1}-y_{1}\right)}{|x-y|^{2}} d \Gamma(y)=a_{12 ; s^{\prime} s} \\
& a_{22 ; s^{\prime} s}=\frac{1}{4 \pi \mu} \int_{\pi_{s^{\prime}}} d \Gamma(x) \int_{\pi_{s}}\left(\log \frac{1}{|x-y|}-\frac{1}{2}+\frac{\left(x_{2}-y_{2}\right)^{2}}{|x-y|^{2}}\right) d \Gamma(y) .
\end{aligned}
$$

Obviously, element-matrix $\left[\begin{array}{ll}a_{11 ; s^{\prime} s} & a_{12 ; s^{\prime} s} \\ a_{21 ; s^{\prime} s} & a_{22 ; s^{\prime} s}\end{array}\right]$ is symmetric.

On the other hand, we get

$$
\begin{aligned}
& a_{11 ; s s^{\prime}}=\frac{1}{4 \pi \mu} \int_{\pi_{s}} d \Gamma(x) \int_{\pi_{s^{\prime}}}\left(\log \frac{1}{|x-y|}-\frac{1}{2}+\frac{\left(x_{1}-y_{1}\right)^{2}}{|x-y|^{2}}\right) d \Gamma(y) \\
& =\frac{1}{4 \pi \mu} \int_{\pi_{s}^{\prime}} d \Gamma(y) \int_{\pi_{s}}\left(\log \frac{1}{|x-y|}-\frac{1}{2}+\frac{\left(x_{1}-y_{1}\right)^{2}}{|x-y|^{2}}\right) d \Gamma(x) \\
& =\frac{1}{4 \pi \mu} \int_{\pi_{s}^{\prime}} d \Gamma(x) \int_{\pi_{s}}\left(\log \frac{1}{|y-x|}-\frac{1}{2}+\frac{\left(y_{1}-x_{1}\right)^{2}}{|y-x|^{2}}\right) d \Gamma(y) \\
& =\frac{1}{4 \pi \mu} \int_{\pi_{s}^{\prime}} d \Gamma(y) \int_{\pi_{s}}\left(\log \frac{1}{|x-y|}-\frac{1}{2}+\frac{\left(x_{1}-y_{1}\right)^{2}}{|x-y|^{2}}\right) d \Gamma(x)=a_{11 ; s^{\prime} s}
\end{aligned}
$$

Similarly, $a_{12 ; s s^{\prime}}=a_{12 ; s^{\prime} s}, a_{21 ; s s^{\prime}}=a_{21 ; s^{\prime} s}, a_{22 ; s s^{\prime}}=a_{22 ; s^{\prime} s}$, therefore

$$
\left[\begin{array}{ll}
a_{11 ; s^{\prime} s} & a_{12 ; s^{\prime} s} \\
a_{21 ; s^{\prime} s} & a_{22 ; s^{\prime} s}
\end{array}\right]=\left[\begin{array}{ll}
a_{11 ; s s^{\prime}} & a_{12 ; s s^{\prime}} \\
a_{21 ; s s^{\prime}} & a_{22 ; s s^{\prime}}
\end{array}\right] .
$$


For $i:=2 s^{\prime}-1, j:=2 s-1, s^{\prime}, s=1, \ldots, N_{1}$, expressing as

$$
\left[\begin{array}{ll}
a_{11 ; s^{\prime} s} & a_{12 ; s^{\prime} s} \\
a_{21 ; s^{\prime} s} & a_{22 ; s^{\prime} s}
\end{array}\right]=:\left[\begin{array}{cc}
a_{i, j} & a_{i, j+1} \\
a_{i+1, j} & a_{i+1, j+1}
\end{array}\right]
$$

we have

$$
\left[\begin{array}{cc}
a_{i, j} & a_{i, j+1} \\
a_{i+1, j} & a_{i+1, j+1}
\end{array}\right]=\left[\begin{array}{cc}
a_{i, j} & a_{i+1, j} \\
a_{i, j+1} & a_{i+1, j+1}
\end{array}\right]=\left[\begin{array}{cc}
a_{i, j} & a_{i, j+1} \\
a_{i+1, j} & a_{i+1, j+1}
\end{array}\right]^{T}
$$

Let us express $V_{h}$-block by $A_{V_{h}}$. Then

$$
A_{V_{h}}=\left(a_{i, j}\right)=\left(a_{j, i}\right)=A_{V_{h}}^{T}
$$

Similarly, symmetry of $D_{h}$-block is established. i.e. $A_{D_{h}}=A_{D_{h}}^{T}$.

Now we will establish block skewsymmetry of blocks $K_{h}^{\prime}$ and $K_{h}$, i.e. $A_{K_{h}^{\prime}}=$ $-A_{K_{h}}^{T}$.

$$
\begin{aligned}
& \left\langle K_{h} \beta_{t} \phi_{t}, w_{s^{\prime}}\right\rangle=\frac{1}{4 \pi} \int_{\Gamma} w_{s^{\prime}}(\varsigma) d \Gamma(\varsigma) \times \\
& \times \int_{\Gamma}\left(\frac{1}{\varsigma-z} n(z) \beta_{t} \phi_{t}(z)+\frac{\varsigma-z}{(\bar{\varsigma}-\bar{z})^{2}} \overline{n(z) \beta_{t} \phi_{t}(z)}\right. \\
& \left.+\frac{1}{\bar{\varsigma}-\bar{z}}\left(n(z) \overline{\beta_{t} \phi_{t}(z)}+\overline{n(z)} \beta_{t} \phi_{t}(z)\right)\right) d \Gamma(z) \\
& =\frac{1}{4 \pi} \int_{\pi_{s^{\prime}}} d \Gamma(\varsigma) \int_{\bar{\pi}_{t}}\left(\frac{1}{\varsigma-z} n(z) \beta_{t} \phi_{t}(z)+\frac{\varsigma-z}{(\bar{\varsigma}-\bar{z})^{2}} \overline{n(z) \beta_{t} \phi_{t}(z)}\right. \\
& \left.+\frac{1}{\bar{\varsigma}-\bar{z}}\left(n(z) \overline{\beta_{t} \phi_{t}(z)}+\overline{n(z)} \beta_{t} \phi_{t}(z)\right)\right) d \Gamma(z) \\
& =\int_{\pi_{s^{\prime}}} d \Gamma(x) \int_{\overline{\bar{\pi}}_{t}}\left(-\frac{1}{\pi r}\right)\left(r_{, i} r_{, j} \beta_{j t} r_{, k} n_{k}(y)\right) \phi_{t}(y) d \Gamma(y) \\
& =\int_{\pi_{s^{\prime}}} d \Gamma(x) \int_{\bar{\pi}_{t}}\left[\begin{array}{ll}
\left(x_{1}-y_{1}\right)\left(x_{1}-y_{1}\right) & \left(x_{2}-y_{2}\right)\left(x_{1}-y_{1}\right) \\
\left(x_{1}-y_{1}\right)\left(x_{2}-y_{2}\right) & \left(x_{2}-y_{2}\right)\left(x_{2}-y_{2}\right)
\end{array}\right]\left[\begin{array}{c}
\beta_{t}^{(1)} \\
\beta_{t}^{(2)}
\end{array}\right] \\
& \times \frac{(x-y) \cdot n(y) \phi_{t}(y)}{\pi|x-y|^{4}} d \Gamma(y)=:\left[\begin{array}{ll}
b_{11 ; s^{\prime} t} & b_{12 ; s^{\prime} t} \\
b_{21 ; s^{\prime} t} & b_{22 ; s^{\prime} t}
\end{array}\right] \text {. }
\end{aligned}
$$

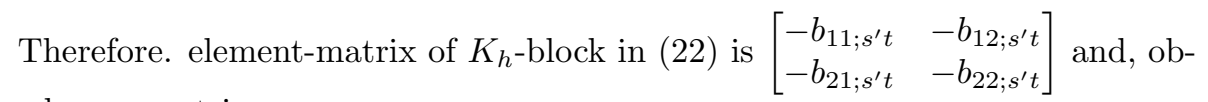
viously, symmetric. 
Similarly, we get for $K_{h}^{\prime}$-block

$$
\begin{aligned}
& \left\langle K_{h}^{\prime} \alpha_{s} w_{s}, \phi_{t^{\prime}}\right\rangle=-\int_{\bar{\pi}_{t^{\prime}}} \phi_{t^{\prime}}(x) d \Gamma(x) \int_{\pi_{s}}\left[\begin{array}{cc}
\left(x_{1}-y_{1}\right)^{2} & \left(x_{1}-y_{1}\right)\left(x_{2}-y_{2}\right) \\
\left(x_{2}-y_{2}\right)\left(x_{1}-y_{1}\right) & \left(x_{2}-y_{2}\right)^{2}
\end{array}\right] \\
& \times \frac{(x-y) \cdot n(x)}{\pi|x-y|^{4}} d \Gamma(y)=:\left[\begin{array}{ll}
a_{11 ; t^{\prime} s} & a_{12 ; t^{\prime} s} \\
a_{21 ; t^{\prime} s} & a_{22 ; t^{\prime} s}
\end{array}\right]\left[\begin{array}{l}
\alpha_{s}^{(1)} \\
\alpha_{s}^{(2)}
\end{array}\right] .
\end{aligned}
$$

Here

$$
\begin{aligned}
& a_{11 ; t^{\prime} s}=-\int_{\bar{\pi}_{t^{\prime}}} \phi_{t^{\prime}}(x) d \Gamma(x) \int_{\pi_{s}}\left(x_{1}-y_{1}\right)^{2} \frac{(x-y) \cdot n(x)}{\pi|x-y|^{4}} d \Gamma(y) \\
& =-\int_{\pi_{s}} d \Gamma(y) \int_{\bar{\pi}_{t^{\prime}}}\left(x_{1}-y_{1}\right)^{2} \frac{(x-y) \cdot n(x)}{\pi|x-y|^{4}} \phi_{t^{\prime}}(x) d \Gamma(x) \\
& =-\int_{\pi_{s}} d \Gamma(x) \int_{\bar{\pi}_{t^{\prime}}}\left(y_{1}-x_{1}\right)^{2} \frac{(y-x) \cdot n(y)}{\pi|y-x|^{4}} \phi_{t^{\prime}}(y) d \Gamma(y) \\
& =\int_{\pi_{s}} d \Gamma(x) \int_{\bar{\pi}_{t^{\prime}}}\left(x_{1}-y_{1}\right)^{2} \frac{(x-y) \cdot n(y)}{\pi|x-y|^{4}} \phi_{t^{\prime}}(y) d \Gamma(y)=b_{11 ; s t^{\prime}},
\end{aligned}
$$

similarly,

$$
a_{12 ; t^{\prime} s}=b_{12 ; s t^{\prime}}, \quad a_{21 ; t^{\prime} s}=b_{21 ; s t^{\prime}}, \quad a_{22 ; t^{\prime} s}=b_{22 ; s t^{\prime}}
$$

Therefore element-matrix

$$
\left[\begin{array}{ll}
a_{11 ; t^{\prime} s} & a_{12 ; t^{\prime} s} \\
a_{21 ; t^{\prime} s} & a_{22 ; t^{\prime} s}
\end{array}\right]=\left[\begin{array}{ll}
b_{11 ; s t^{\prime}} & b_{12 ; s t^{\prime}} \\
b_{21 ; s t^{\prime}} & b_{22 ; s t^{\prime}}
\end{array}\right]
$$

is symmetric.

We denote for $i=2 s^{\prime}-1, j=2 N_{1}+2 t-1, s=1, \ldots, N_{1}, t=1, \ldots, N_{2}$

$$
\left[\begin{array}{ll}
-b_{11 ; s^{\prime} t} & -b_{12 ; s^{\prime} t} \\
-b_{21 ; s^{\prime} t} & -b_{22 ; s^{\prime} t}
\end{array}\right]=:\left[\begin{array}{cc}
a_{i, j} & a_{i, j+1} \\
a_{i+1, j} & a_{i+1, j+1}
\end{array}\right]
$$

and for $i^{\prime}=2 N_{1}+2 t^{\prime}-1, j^{\prime}=2 s-1, s=1, \ldots, N_{1}, t^{\prime}=1, \ldots, N_{2}$

$$
\left[\begin{array}{ll}
a_{11 ; t^{\prime} s} & a_{12 ; t^{\prime} s} \\
a_{21 ; t^{\prime} s} & a_{22 ; t^{\prime} s}
\end{array}\right]=:\left[\begin{array}{cc}
a_{i^{\prime}, j^{\prime}} & a_{i^{\prime}, j^{\prime}+1} \\
a_{i^{\prime}+1, j^{\prime}} & a_{i^{\prime}+1, j^{\prime}+1}
\end{array}\right] .
$$

Then element-matrices (a1) and (a2) are symmetric, respectively, moreover if $s^{\prime}=s, t=t^{\prime}$ then $i=2 s-1=j^{\prime}, j=2 N_{1}+2 t^{\prime}-1=i^{\prime}$ and thus

$$
\left[\begin{array}{ll}
b_{11 ; s t^{\prime}} & b_{12 ; s t^{\prime}} \\
b_{21 ; s t^{\prime}} & b_{22 ; s t^{\prime}}
\end{array}\right]=\left[\begin{array}{ll}
b_{11 ; s^{\prime} t} & b_{12 ; s^{\prime} t} \\
b_{21 ; s^{\prime} t} & b_{22 ; s^{\prime} t}
\end{array}\right]=\left[\begin{array}{cc}
-a_{i, j} & -a_{i, j+1} \\
-a_{i+1, j} & -a_{i+1, j+1}
\end{array}\right]
$$




$$
\left[\begin{array}{cc}
a_{11 ; t^{\prime} s} & a_{12 ; t^{\prime} s} \\
a_{21 ; t^{\prime} s} & a_{22 ; t^{\prime} s}
\end{array}\right]=\left[\begin{array}{cc}
a_{i^{\prime}, j^{\prime}} & a_{i^{\prime}, j^{\prime}+1} \\
a_{i^{\prime}+1, j^{\prime}} & a_{i^{\prime}+1, j^{\prime}+1}
\end{array}\right]=\left[\begin{array}{cc}
a_{j, i} & a_{j, i+1} \\
a_{j+1, i} & a_{j+1, i+1}
\end{array}\right]
$$

For all $i, j$ combining element-matrices $\left[\begin{array}{cc}-a_{i, j} & -a_{i, j+1} \\ -a_{i+1, j} & -a_{i+1, j+1}\end{array}\right]$ we get block $\left(-A_{K_{h}}\right)$, and combining element-matrices $\left[\begin{array}{cc}a_{j, i} & a_{j, i+1} \\ a_{j+1, i} & a_{j+1, i+1}\end{array}\right]$ block $\left(A_{K_{h}^{\prime}}^{T}\right)$. From (a2) we have $A_{K^{\prime}}=-A_{K}^{T}$.

Collecting results we have that in the real coefficient matrix $A:=\left[\begin{array}{ll}A_{V_{h}} & A_{K_{h}} \\ A_{K_{h}^{\prime}} & A_{D_{h}}\end{array}\right]$ blocks $A_{V_{h}}$ and $A_{D_{h}}$ are, respectively, symmetric, and $A_{K_{h}}$ and $A_{K_{h}^{\prime}}$ are block skewsymmetric.

\section{A3. Explain on Mapping properties of the boundary integral Opera-} tors

For Stokes flow problem it was proved that mappings of the boundary integral operators

$$
V: H^{-1 / 2}(\Gamma) \rightarrow H^{1 / 2}(\Gamma), K: H^{1 / 2}(\Gamma) \rightarrow H^{1 / 2}(\Gamma), D: H^{1 / 2}(\Gamma) \rightarrow H^{-1 / 2}(\Gamma)
$$

are continuous(cf. [11], Lemma 5.6.4, where the operators $V, K, D$ are denoted $\gamma_{0} V, \gamma_{0} W, D$, respectively). For elastostatic problem which correspond to the Stokes problem when the Poisson ratio tends to $1 / 2$, it holds that $K^{\prime}: H^{-1 / 2}(\Gamma) \rightarrow$ $H^{-1 / 2}(\Gamma)$ is continuous(cf. [6], Theorem 2 for $s=0$ ). These properties are obtained by using solvability and regularity of associated transmission problems(cf. [11], 5.6.2). Note that for the potential problem also above properties are held. In the case of 2-D potential problem we explain only for simple layer boundary integral operator $V$, the integral kernel of which is $\frac{1}{\pi} \log \frac{1}{|x-y|}$ (refer to [2] Chapter 7). Denoting by $C$ the circumstance of unit circle about the origin, let us introduce $2 \pi$ periodic parameter as $x=(\cos t, \sin t), y=(\cos s, \sin s),(x, y) \in C, t, s \in[0,2 \pi]$. Now, choose arbitrary density $\sigma(y) \in L^{2}(C)$. Then $\sigma(y)=\sigma((\cos s, \sin s))=: \varphi(s)$ and

$$
\begin{aligned}
& V \sigma(x)=V \varphi(t)=\frac{1}{2 \pi} \int_{0}^{2 \pi} \log \frac{1}{\left|(\cos t-\cos s)^{2}+\left(\sin t-\left.\sin s\right|^{2}\right)\right|^{1 / 2}} \varphi(s) d s \\
& =\frac{1}{\pi} \int_{0}^{2 \pi} \log \frac{1}{\left|\sin ^{2} \frac{t-s}{2}\right|^{1 / 2}} \varphi(s) d s=\frac{1}{\pi} \int_{0}^{2 \pi} \log \frac{2 e^{-1 / 2}}{\left|2 e^{-1 / 2} \sin \frac{t-s}{2}\right|} \varphi(s) d s \\
& =\frac{1}{\pi} \int_{0}^{2 \pi} \log \frac{1}{\left|2 e^{-1 / 2} \sin \frac{t-s}{2}\right|} \varphi(s) d s+\frac{1}{\pi} \int_{0}^{2 \pi}(\log (2)-1 / 2) \varphi(s) d s \\
& =: V_{0} \varphi(s)+V_{1} \varphi(s),
\end{aligned}
$$


where $V_{0}$ is principal operator and, obviously, $V_{0} \varphi$ and $V_{1} \varphi$ are ,respectively, $2 \pi-$ periodic functions and belong to $L^{2}(0,2 \pi)$.

For any real number $r \geq 0$, define $H^{r}(2 \pi)$ to be the set of all $2 \pi$ - periodic functions $\varphi \in L^{2}(0,2 \pi)$ for which

$$
\|\varphi\|_{r}=\frac{1}{\sqrt{2 \pi}}\left[|\hat{\varphi}(0)|^{2}+\sum_{|m|>0}|m|^{2 r}|\hat{\varphi}(m)|^{2}\right]^{1 / 2}<\infty,
$$

where $\hat{\varphi}(m)$ is Fourier coefficient of $\varphi(s)$, that is

$$
\hat{\varphi}(m)=\frac{1}{\sqrt{2 \pi}} \int_{0}^{2 \pi} \varphi(s) e^{-i m s} d s .
$$

It holds (cf. [2],(7.2.74)) that

$$
V_{0} \varphi(t)=\frac{1}{\sqrt{2 \pi}}\left(\hat{\varphi}(0)+\sum_{|m|>0} \frac{\hat{\varphi}(m)}{|m|} e^{i m t}\right) .
$$

Then, for $r \geq 0$ it holds that

$$
\begin{aligned}
& \left\|V_{0} \varphi\right\|_{r+1}=\frac{1}{\sqrt{2 \pi}}\left[|\hat{\varphi}(0)|^{2}+\sum_{|m|>0}|m|^{2 r+2}\left|\frac{\hat{\varphi}(m)}{|m|}\right|^{2}\right]^{1 / 2} \\
& =\frac{1}{\sqrt{2 \pi}}\left[|\hat{\varphi}(0)|^{2}+\sum_{|m|>0}|m|^{2 r}|\hat{\varphi}(m)|^{2}\right]^{1 / 2}=\|\varphi\|_{r},
\end{aligned}
$$

which implies that

$$
V_{0}: H^{r}(2 \pi) \rightarrow H^{r+1}(2 \pi)-\text { isomorphism. }
$$

This estimate is extended to allow $r<0$ by duality, therefor, for $r=-1 / 2$ give

$$
V_{0}: H^{-1 / 2}(2 \pi) \rightarrow H^{1 / 2}(2 \pi),
$$

and, taking account of $V_{1} \varphi=$ const,

$$
V: H^{-1 / 2}(2 \pi) \rightarrow H^{1 / 2}(2 \pi) .
$$

This result lead to

$$
V: H^{-1 / 2}(C) \rightarrow H^{1 / 2}(C),
$$

moreover, for smooth boundary $\Gamma$

$$
V: H^{-1 / 2}(\Gamma) \rightarrow H^{1 / 2}(\Gamma)
$$


which all are continuous.

\section{A4. Calculation of hypersingular integrals}

A4.1. Calculation of matrix element $\left\langle D_{h} \beta_{t} \phi_{t}, \phi_{t^{\prime}}\right\rangle, t=t^{\prime}$

First we calculate the integrals $I_{1}\left(\beta_{t}\right), I_{2}\left(\beta_{t}\right)$.

$$
\begin{aligned}
& I_{1}\left(\beta_{t}\right)= \\
& =\beta_{t} \int_{z_{t-1}}^{z_{t}} \phi_{t}(\varsigma) d \Gamma(\varsigma) \int_{z_{t-1}}^{z_{t}}\left(\frac{1}{(\varsigma-z)^{2}} n(z) n(\varsigma)+\frac{1}{(\bar{\varsigma}-\bar{z})^{2}} \overline{n(z) n(\varsigma)}\right) \phi_{t}(z) d \Gamma(z) .
\end{aligned}
$$

By (32)-(34) in subsection 3.2 we have

$$
\begin{aligned}
& z=z_{t-1}+\xi e_{t-1}, \phi_{t}(z)=\xi, n(z)=-i e_{t-1} / L_{t-1}, d \Gamma(z)=L_{t-1} d \xi \\
& \varsigma=z_{t-1}+\eta e_{t-1}, \phi_{t}(\varsigma)=\eta, n(\varsigma)=-i e_{t-1} / L_{t-1}, d \Gamma(\varsigma)=L_{t-1} d \eta
\end{aligned}
$$

Now, let us apply boundary limit method for calculation of $I_{1}\left(\beta_{t}\right)$. Taking a point $\varsigma_{\varepsilon}=\varsigma+\varepsilon L_{t-1} n(\varsigma)$ apart $\varsigma$, by variable change (a4) we have

$$
\begin{aligned}
& \varsigma_{\varepsilon}=z_{t-1}+\eta e_{t-1}+\varepsilon L_{t-1}\left(-i e_{t-1} / L_{t-1}\right)=z_{t-1}+(\eta-i \varepsilon) e_{t-1}, \\
& \varsigma_{\varepsilon}-z=z_{t-1}+(\eta-i \varepsilon) e_{t-1}-\left(z_{t-1}+\xi e_{t-1}\right)=\xi e_{t-1}+(\eta-i \varepsilon) e_{t-1} .
\end{aligned}
$$

Denote by $I_{1}^{\varepsilon}\left(\beta_{t}\right)$ the regular integral obtained by substituting $\varsigma_{\varepsilon}-z$ instead of $\varsigma-z$ in integral $I_{1}\left(\beta_{t}\right)$ of (a3), that is,

$$
\begin{aligned}
& I_{1}^{\varepsilon}\left(\beta_{t}\right)= \\
& \beta_{t} \int_{z_{t-1}}^{z_{t}} \phi_{t}(\varsigma) d \Gamma(\varsigma) \int_{z_{t-1}}^{z_{t}}\left(\frac{1}{\left(\varsigma_{\varepsilon}-z\right)^{2}} n(z) n(\varsigma)+\frac{1}{\left(\bar{\varsigma}_{\varepsilon}-\bar{z}\right)^{2}} \overline{n(z) n(\varsigma)}\right) \phi_{t}(z) d \Gamma(z) .
\end{aligned}
$$


Then, by (a4) we have

$$
\begin{aligned}
& I_{1}^{\varepsilon}\left(\beta_{t}\right)=\beta_{t} \int_{z_{t-1}}^{z_{t}} \eta L_{t-1} d \eta \times \\
& \times \int_{z_{t-1}}^{z_{t}}\left(\frac{-e_{t-1} e_{t-1} /\left(L_{t-1} L_{t-1}\right)}{\left((\eta-i \varepsilon-\xi) e_{t-1}\right)^{2}}+\frac{-\bar{e}_{t-1} \bar{e}_{t-1} /\left(L_{t-1} L_{t-1}\right)}{\left((\eta+i \varepsilon-\xi) \bar{e}_{t-1}\right)^{2}}\right) \xi L_{t-1} d \xi \\
& =\beta_{t}(-1) \int_{0}^{1} \eta d \eta \int_{0}^{1} \xi d \xi\left(\frac{1}{(\eta-i \varepsilon-\xi)^{2}}+\frac{1}{(\eta+i \varepsilon-\xi)^{2}}\right) \\
& =\beta_{t}(-1)\left(1-\left(-1+(i \varepsilon)^{2}\right) \log \left(-(i \varepsilon)^{2}\right)+\left(-1+(i \varepsilon)^{2}\right) \log \left(1-(i \varepsilon)^{2}\right)\right) \\
& =\beta_{t}(-1)\left(1-\left(-1-\varepsilon^{2}\right) \log \left(\varepsilon^{2}\right)+\left(-1-\varepsilon^{2}\right) \log \left(1+\varepsilon^{2}\right)\right) .
\end{aligned}
$$

The limit as $\varepsilon \rightarrow 0$ is

$$
I_{1}\left(\beta_{t}\right)=-\beta_{t}-\beta_{t} \log \left(\varepsilon^{2}\right),
$$

where $-\log \left(\varepsilon^{2}\right)$ is divergent term.

By the same way we can calculate $I_{i}\left(\beta_{t}\right), i=2,3,4$.

$$
\begin{aligned}
& I_{2}\left(\beta_{t}\right)=\beta_{t} \frac{1}{2}\left(2-\frac{e_{t-1}}{e_{t}} \log \left(e_{t-1}\right)-\left(2+\frac{e_{t}}{e_{t-1}}\right) \log \left(e_{t}\right)\right. \\
& +\left(2+\frac{e_{t-1}}{e_{t}}+\frac{e_{t}}{e_{t-1}}\right) \log \left(e_{t-1}+e_{t}\right)-\frac{\bar{e}_{t-1}}{\bar{e}_{t}} \log \left(\bar{e}_{t-1}\right) \\
& \left.-\left(2+\frac{\bar{e}_{t}}{\bar{e}_{t-1}}\right) \log \left(\bar{e}_{t}\right)+\left(2+\frac{\bar{e}_{t-1}}{\bar{e}_{t}}+\frac{\bar{e}_{t}}{\bar{e}_{t-1}}\right) \log \left(\bar{e}_{t-1}+\bar{e}_{t}\right)\right)+\beta_{t} \log \left(\varepsilon^{2}\right)
\end{aligned}
$$

which is sum of the finite integral value and divergent term $\beta_{t} \log \left(\varepsilon^{2}\right)$.

$$
\begin{aligned}
& I_{3}\left(\beta_{t}\right)=\beta_{t} \frac{1}{2}\left(2-\frac{e_{t}}{e_{t-1}} \log \left(e_{t}\right)-\left(2+\frac{e_{t-1}}{e_{t}}\right) \log \left(e_{t-1}\right)\right. \\
& +\left(2+\frac{e_{t-1}}{e_{t}}+\frac{e_{t}}{e_{t-1}}\right) \log \left(e_{t}+e_{t-1}\right)-\frac{\bar{e}_{t}}{\bar{e}_{t-1}} \log \left(\bar{e}_{t}\right)-\left(2+\frac{\bar{e}_{t-1}}{\bar{e}_{t}}\right) \log \left(\bar{e}_{t-1}\right) \\
& \left.+\left(2+\frac{\bar{e}_{t-1}}{\bar{e}_{t}}+\frac{\bar{e}_{t}}{\bar{e}_{t-1}}\right) \log \left(\bar{e}_{t}+\bar{e}_{t-1}\right)\right)+\beta_{t} \log \left(\varepsilon^{2}\right), \\
& I_{4}\left(\beta_{t}\right)=\beta_{t}(-1)-\beta_{t} \log \left(\varepsilon^{2}\right) .
\end{aligned}
$$


Next, we calculate $I_{k}\left(\bar{\beta}_{t}\right), k=1,2,3,4$.

$$
\begin{aligned}
& I_{1}\left(\bar{\beta}_{t}\right)=\bar{\beta}_{t} \int_{z_{t-1}}^{z_{t}} \phi_{t} \times(\varsigma) d \Gamma(\varsigma) \\
& \times \int_{z_{t-1}}^{z_{t}}\left(\frac{\overline{n(z)} n(\varsigma)+n(z) \overline{n(\varsigma)}}{(\bar{\varsigma}-\bar{z})^{2}}+\frac{2(\varsigma-z)}{(\bar{\varsigma}-\bar{z})^{3}} \overline{n(z) n(\varsigma)}\right) \overline{\phi_{t}(z)} d \Gamma(z) \\
& =\bar{\beta}_{t} \int_{0}^{1} \eta L_{t-1} d \eta \times \int_{0}^{1}\left(\frac{1}{\left((\eta-\xi) \bar{e}_{t-1}\right)^{2}}\left(\frac{\bar{e}_{t-1} e_{t-1}}{L_{t-1} L_{t-1}}+\frac{e_{t-1} \bar{e}_{t-1}}{L_{t-1} L_{t-1}}\right)\right. \\
& \left.+\frac{2(\eta-\xi) e_{t-1}}{\left((\eta-\xi) \bar{e}_{t-1}\right)^{3}} \frac{-\bar{e}_{t-1} \bar{e}_{t-1}}{L_{t-1} L_{t-1}}\right) \xi L_{t-1} d \xi \\
& =\bar{\beta}_{t} \int_{0}^{1} \eta d \eta \int_{0}^{1}\left(\frac{e_{t-1} / \bar{e}_{t-1}+e_{t-1} / \bar{e}_{t-1}}{(\eta-\xi)^{2}}-\frac{2(\eta-\xi) e_{t-1} / \bar{e}_{t-1}}{(\eta-\xi)^{3}}\right) \xi d \xi=0 . \\
& I_{2}\left(\bar{\beta}_{t}\right)=\bar{\beta}_{t} \frac{1}{2}\left(3 \frac{e_{t-1}}{\bar{e}_{t-1}}+\frac{e_{t}}{\bar{e}_{t}}-\left(\frac{e_{t} \bar{e}_{t-1}}{\bar{e}_{t}^{2}}-\frac{e_{t-1}}{\bar{e}_{t}}\right) \log \left(\bar{e}_{t-1}\right)\right. \\
& +\left(\frac{e_{t}}{\bar{e}_{t-1}}-\frac{e_{t-1} \bar{e}_{t}}{\bar{e}_{t-1}^{2}}\right) \log \left(\bar{e}_{t}\right) \\
& \left.+\left(\frac{e_{t} \bar{e}_{t-1}}{\bar{e}_{t}^{2}}-\frac{e_{t-1}}{\bar{e}_{t}}-\frac{e_{t}}{\bar{e}_{t-1}}+\frac{e_{t-1} \bar{e}_{t}}{\bar{e}_{t-1}^{2}}\right) \log \left(\bar{e}_{t-1}+\bar{e}_{t}\right)\right) \text {. } \\
& I_{3}\left(\bar{\beta}_{t}\right)=\bar{\beta}_{t} \frac{1}{2}\left(3 \frac{e_{t}}{\bar{e}_{t}}+\frac{e_{t-1}}{\bar{e}_{t-1}}-\left(\frac{e_{t-1} \bar{e}_{t}}{\bar{e}_{t-1}^{2}}-\frac{e_{t}}{\bar{e}_{t-1}}\right) \log \left(\bar{e}_{t}\right)\right. \\
& +\left(\frac{e_{t-1}}{\bar{e}_{t}}-\frac{e_{t} \bar{e}_{t-1}}{\bar{e}_{t}^{2}}\right) \log \left(\bar{e}_{t-1}\right) \\
& \left.+\left(\frac{e_{t-1} \bar{e}_{t}}{\bar{e}_{t-1}^{2}}-\frac{e_{t}}{\bar{e}_{t-1}}-\frac{e_{t-1}}{\bar{e}_{t}}+\frac{e_{t} \bar{e}_{t-1}}{\bar{e}_{t}^{2}}\right) \log \left(\bar{e}_{t}+\bar{e}_{t-1}\right)\right), \\
& I_{4}\left(\bar{\beta}_{t}\right)=0 .
\end{aligned}
$$

A.4.2. calculation of matrix element $\left\langle D_{h} \beta_{t} \phi_{t}, \phi_{t^{\prime}}\right\rangle,\left|t-t^{\prime}\right|=1$

Among integrals in (43), $I_{2}\left(\beta_{t}\right)$ and $I_{2}\left(\bar{\beta}_{t}\right)$, being element-coincident integral, may contain divergent terms. Calculating $I_{2}\left(\beta_{t}\right)$ by the boundary limit method, 
we get

$$
\begin{aligned}
& I_{2}^{\varepsilon}\left(\beta_{t}\right)= \\
& =\beta_{t} \int_{z_{t}}^{z_{t+1}} \phi_{t+1}(\varsigma) d \Gamma(\varsigma) \int_{z_{t}}^{z_{t+1}}\left(\frac{1}{\left(\varsigma_{\varepsilon}-z\right)^{2}} n(z) n(\varsigma)+\frac{1}{\left(\bar{\varsigma}_{\varepsilon}-\bar{z}\right)^{2}} \overline{n(z) n(\varsigma)}\right) \phi_{t}(z) d \Gamma(z) \\
& =\beta_{t} \int_{0}^{1} \eta d \eta \int_{0}^{1}\left(\frac{-1}{(\eta-i \varepsilon-\xi)^{2}}+\frac{-1}{(\eta+i \varepsilon-\xi)^{2}}\right)(1-\xi) d \xi \\
& =\beta_{t} \frac{1}{2}\left(2-\log \left(-(i \varepsilon)^{2}\right)-\left(-1+(i \varepsilon)^{2}\right) \log \left(-(i \varepsilon)^{2}\right)\right. \\
& +\log \left(1-(i \varepsilon)^{2}\right)+(i \varepsilon)^{2} \log \left(1-(i \varepsilon)^{2}\right)-(i \varepsilon)^{2} \log \left(-(i \varepsilon)^{2}\right) \\
& \left.+\left(-1+(i \varepsilon)^{2}\right) \log \left(1-(i \varepsilon)^{2}\right)\right)
\end{aligned}
$$

Here, $-\log \left(-(i \varepsilon)^{2}\right)=-\log \left(\varepsilon^{2}\right)$ is divergent term and

$$
-\left(-1+(i \varepsilon)^{2}\right) \log \left(-(i \varepsilon)^{2}\right)=+\left(1+\varepsilon^{2}\right) \log \left(\varepsilon^{2}\right) \rightarrow+\log \left(\varepsilon^{2}\right)(\varepsilon \rightarrow 0)
$$

is also divergent term, but these divergent terms cancel each other out. Also,

$$
\begin{aligned}
& -(i \varepsilon)^{2} \log \left(-(i \varepsilon)^{2}\right)=\varepsilon^{2} \log \left(\varepsilon^{2}\right) \rightarrow 0 \text { as } \varepsilon \rightarrow 0, \\
& \log \left(1-(i \varepsilon)^{2}\right)+(i \varepsilon)^{2} \log \left(1-(i \varepsilon)^{2}\right)+\left(-1+(i \varepsilon)^{2}\right) \log \left(1-(i \varepsilon)^{2}\right) \\
& =\log \left(1+\varepsilon^{2}\right)-\varepsilon^{2} \log \left(1+\varepsilon^{2}\right)+\left(-1-\varepsilon^{2}\right) \log \left(1+\varepsilon^{2}\right) \rightarrow 0, \text { as } \varepsilon \rightarrow 0 .
\end{aligned}
$$

Therefore, we get that $I_{2}\left(\beta_{t}\right)=\lim _{\varepsilon \rightarrow 0} I_{2}^{\varepsilon}\left(\beta_{t}\right)=\beta_{t}$.

$I_{2}\left(\bar{\beta}_{t}\right)$ is element-coincident integral too, but have no divergent term as former subsection. Other element integrals are weak singular because their interior and exterior integral intervals intersect only at end point. We see $I_{1}\left(\beta_{t}\right)$ only.

$$
\begin{aligned}
& I_{1}\left(\beta_{t}\right)=\beta_{t} \int_{z_{t}}^{z_{t+1}} \phi_{t+1}(\varsigma) d \Gamma(\varsigma) \int_{z_{t-1}}^{z_{t}}\left(\frac{n(z) n(\varsigma)}{(\varsigma-)^{2}}+\frac{\overline{n(z) n(\varsigma)}}{(\bar{\varsigma}-\bar{z})^{2}}\right) \phi_{t}(z) d \Gamma(z) \\
& =\beta_{t} \int_{0}^{1} \eta d \eta \int_{0}^{1}\left(\frac{-e_{t} e_{t-1}}{\left(\eta e_{t}+(1-\xi) e_{t-1}\right)^{2}}+\frac{-\bar{e}_{t} \bar{e}_{t-1}}{\left(\eta \bar{e}_{t}+(1-\xi) \bar{e}_{t-1}\right)^{2}}\right) \xi d \xi \\
& =\beta_{t} \frac{1}{2}\left(-2-\frac{e_{t}}{e_{t-1}} \log \left(e_{t}\right)+\left(\frac{e_{t}}{e_{t-1}}-\frac{e_{t-1}}{e_{t}}\right) \log \left(e_{t}+e_{t-1}\right)-\frac{\bar{e}_{t}}{\bar{e}_{t-1}} \log \left(\bar{e}_{t}\right)\right. \\
& +\left(\frac{\bar{e}_{t}}{\bar{e}_{t-1}}-\frac{\bar{e}_{t-1}}{\bar{e}_{t}}\right) \log \left(\bar{e}_{t}+\bar{e}_{t-1}\right)+\frac{e_{t-1}}{e_{t}} \log \left(e_{t-1}\right)+\frac{\bar{e}_{t-1}}{\bar{e}_{t}} \log \left(\bar{e}_{t-1}\right) \\
& \left.+\left(\frac{\eta}{e_{t-1}}\left(\eta e_{t}\right) \log \left(-\eta e_{t}\right)+\frac{\eta}{\bar{e}_{t-1}}\left(\eta \bar{e}_{t}\right) \log \left(-\eta \bar{e}_{t}\right)\right)_{\eta=0}\right)
\end{aligned}
$$




$$
\begin{aligned}
& \text { Since }\left(\frac{\eta}{e_{t-1}}\left(\eta e_{t}\right) \log \left(-\eta e_{t}\right)+\frac{\eta}{\bar{e}_{t-1}}\left(\eta \bar{e}_{t}\right) \log \left(-\eta \bar{e}_{t}\right)\right)_{\eta=0}=0 \text {, we get } \\
& I_{1}\left(\beta_{t}\right)=\beta_{t} \frac{1}{2}\left(-2-\frac{e_{t}}{e_{t-1}} \log \left(e_{t}\right)+\frac{e_{t-1}}{e_{t}} \log \left(e_{t-1}\right)-\frac{\bar{e}_{t}}{\bar{e}_{t-1}} \log \left(\bar{e}_{t}\right)\right. \\
& +\frac{\bar{e}_{t-1}}{\bar{e}_{t}} \log \left(\bar{e}_{t-1}\right)+\left(\frac{e_{t}}{e_{t-1}}-\frac{e_{t-1}}{e_{t}}\right) \log \left(e_{t}+e_{t-1}\right) \\
& \left.+\left(\frac{\bar{e}_{t}}{\bar{e}_{t-1}}-\frac{\bar{e}_{t-1}}{\bar{e}_{t}}\right) \log \left(\bar{e}_{t}+\bar{e}_{t-1}\right)\right) .
\end{aligned}
$$

\section{References}

[1] Aimi, A., Diligenti, M., Hypersingular kernel integration in 3D Galerkin boundary element method, Journal of Computational and Applied Mathematics 138(2002), 51 - 72 .

[2] Atkinson, K., The Numerical Solution of Integral Equations of the Second Kind, Cambridge University Press, 1997

[3] Bakar, S. A., Saleh, A. L., A technique to remove second order singularity application in the boundary element method for elastoplasticity plane stress analysis, Electronic Journal of Boundary Elements, 8(1)(2010), 1 - 9.

[4] Beylkin, G., Cramer, A., A multiresolution approach to regularization of singular operators and fast summation, SIAM J. Sci. Comput., 24(1)(2002), 81 - 117.

[5] Cho, M. H., Cai, W., A wideband fast multipole method for the twodimensional complex Helmholtz equation, Computer Physics Communications, 1812010,2086 - 2090.

[6] Das, R., Hota, M. K. and Bej, M., Some derivative-free quadrature rules for numerical approximations of Cauchy principal value of integrals, Hindawi Publishing Corporation ISRN, Computational Mathematics, Volume 2014, Article ID $1863971-8$.

[7] Eck, C., Steinbach, O. and Wendland, W. L., A symmetric boundary element method for contact problems with friction, Mathematics and Computers in Simulation, 50(1999), 43 - 61.

[8] Emmanuel, B., A complex parameter boundary element method for modeling AC impedances of electrochemical systems by analytic continuation- Application to a slit geometry, Engineering Analysis with Boundary Elements, 32(2008), $770-777$. 
[9] Fedotov, V. P., Spevak, L. F., One approach to the derivation of exact integration formulae in the boundary element method, Engineering Analysis with Boundary Elements, 32(2008), 883 - 888.

[10] Grecu, L., Grecu, V., Demian, M. and Demian, G., A complex variable boundary element method for the flow around obstacles, Proceedings of the World Congress on Engineering 2009, Vol II, WCE 2009, July 1 - 3, London, U.K., 2009.

[11] Hsiao, G. C., Wendland, W. L., Boundary Integral Equations, Springer, 2008.

[12] Liu, Y., Chen, S., A new form of the hypersingular boundary integral equation for 3-D acoustics and its implementation with Cboundary elements, Comput. Meth. Appl. Mech. Engrg., 173(1999), 375 - 386.

[13] Liu, Y. J., Nishimura, N. and Yao, Z. H., A fast multipole accelerated method of fundamental solutions for potential problems, Engineering Analysis with Boundary Elements, 29(2005), 1016 - 1024.

[14] Liu, Y. J., A new fast multipole boundary element method for solving 2-D Stokes flow problems based on a dual BIE formulation, Engineering Analysis with Boundary Elements, 32(2008), 139 - 151.

[15] Liu, Y. J., A new fast multipole boundary element method for solving large scale two-dimentional elastostatic problems, International Journal for $\mathrm{Nu}$ merical Methods in Engineering, 65(6)(2005), 863 - 881.

[16] Nesvit, K. V., Scattering of TE Wave on Screened Pre-Cantor Grating Based on Hypersingular Integral Equations, American Journal of Computational and Applied Mathematics, 4(1)(2014), 9 - 16.

[17] Of, G., Steinbach, O. and Wendland, W. L., The fast multipole boundary element method for the symmetric boundary integral formulation, IABEM 2002, International Association for Boundary Element Methods, UT Austin, TX, USA, May 28-30, 2002.

[18] Panzecaa, T., Cucco, F. and Terravecchia, S., Symmetric boundary element method versus finite element method, Comput. Methods Appl. Mech. Engrg. 191(2002), 3347-3367.

[19] Sutradhar, A., Paulino, G. H. and Gray, L. T., Symmetric Galerkin boundary element method, Springer, 2008.

[20] Sato, K., Complex variable boundary element method for potential flow with thin objects, Comput. Methods Appl. Mech. Engrg., 192(2003), 1421 - 1433.

[21] Vladimirescu, I., Grecu, L., Weakening the Singularities when applying the BEM for 2D compressible fluid flow, Proccedings of the International MultiConferece of Engineers and Computer Scientists 2010: Vol III,IMECS 2010, March 17-19, Hong Kong, 2010. 